\title{
Search for neutral Higgs bosons in the minimal supersymmetric extension of the standard model
}

\author{
OPAL Collaboration
}

R. Akers ${ }^{16}$, G. Alexander ${ }^{23}$, J. Allison ${ }^{16}$, K.J. Anderson ${ }^{9}$, S. Arcelli ${ }^{2}$, S. Asai ${ }^{24}$, A. Astbury ${ }^{28}$, D. Axen ${ }^{29}$, G. Azuelos ${ }^{18, a}$, A.H. Ball ${ }^{17}$, E. Barberio ${ }^{26}$, R.J. Barlow ${ }^{16}$, R. Bartoldus ${ }^{3}$, J.R. Batley ${ }^{5}$, G. Beaudoin ${ }^{18}$, A. Beck ${ }^{23}$, G.A. Beck ${ }^{13}$, J. Becker ${ }^{10}$, C. Beeston ${ }^{16}$, T. Behnke ${ }^{27}$, K.W.Bell ${ }^{20}$, G. Bella ${ }^{23}$, P.Bentkowski ${ }^{18}$, S. Bentvelsen ${ }^{8}$, P.Berlich ${ }^{10}$, S. Bethke ${ }^{32}$, O. Biebel ${ }^{32}$, I.J. Bloodworth ${ }^{1}$, P.Bock ${ }^{11}$, H.M. Bosch ${ }^{11}$, M. Boutemeur ${ }^{18}$, S. Braibant ${ }^{12}$, P. Bright-Thomas ${ }^{25}$, R.M. Brown ${ }^{20}$, A. Buijs ${ }^{8}$, H.J. Burckhart ${ }^{8}$, C. Burgard ${ }^{27}$, P. Capiluppi ${ }^{2}$, R.K. Carnegie ${ }^{6}$, A.A. Carter ${ }^{13}$, J.R. Carter ${ }^{5}$, C.Y. Chang ${ }^{17}$, C. Charlesworth ${ }^{6}$, D.G. Charlton ${ }^{8}$, S.L. Chu ${ }^{4}$, P.E.L.Clarke ${ }^{15}$, J.C.Clayton ${ }^{1}$, S.G.Clowes ${ }^{16}$, I. Cohen ${ }^{23}$, J.E.Conboy ${ }^{15}$, M. Coupland ${ }^{14}$, M.Cuffiani ${ }^{2}$, S. Dado ${ }^{22}$, C. Dallapiccola ${ }^{17}$, G.M.Dallavalle ${ }^{2}$, C. Darling ${ }^{31}$, S. De Jong ${ }^{13}$, H. Deng ${ }^{17}$, M. Dittmar ${ }^{4}$, M.S. Dixit ${ }^{7}$, E. do Couto e Silva ${ }^{12}$, J.E. Duboscq ${ }^{8}$, E. Duchovni ${ }^{26}$, G. Duckeck ${ }^{8}$, I.P. Duerdoth ${ }^{16}$, U.C. Dunwoody ${ }^{5}$, P.A. Elcombe ${ }^{5}$, P.G. Estabrooks ${ }^{6}$, E. Etzion ${ }^{23}$, H.G. Evans ${ }^{9}$, F. Fabbri ${ }^{2}$, B. Fabbro ${ }^{21}$, M. Fanti ${ }^{2}$, M. Fierro ${ }^{2}$, M. Fincke-Keeler ${ }^{28}$, H.M. Fischer ${ }^{3}$, P.Fischer ${ }^{3}$, R. Folman ${ }^{26}$, D.G. Fong ${ }^{17}$, M. Foucher ${ }^{17}$, H. Fukui ${ }^{24}$, A. Fürtjes ${ }^{8}$, P. Gagnon ${ }^{6}$, A. Gaidot ${ }^{21}$, O. Ganel ${ }^{26, b}$, J.W. Gary ${ }^{4}$, J. Gascon ${ }^{18}$, N.I. Geddes ${ }^{20}$, C. Geich-Gimbel ${ }^{3}$, S.W.Gensler ${ }^{9}$, F.X. Gentit ${ }^{21}$, T. Geralis ${ }^{20}$, G. Giacomelli ${ }^{2}$, P. Giacomelli ${ }^{4}$, R. Giacomelli2 ${ }^{2}$, V. Gibson ${ }^{5}$, W.R. Gibson ${ }^{13}$, J.D. Gillies ${ }^{20}$, J. Goldberg ${ }^{22}$, D.M. Gingrich ${ }^{30, a}$, M.J. Goodrick ${ }^{5}$, W. Gorn ${ }^{4}$, C. Grandi ${ }^{2}$, P. Grannis ${ }^{8}$, E. Gross ${ }^{26}$, J.Hagemann ${ }^{27}$, G.G. Hanson ${ }^{12}$, M. Hansroul ${ }^{8}$, C.K. Hargrove ${ }^{7}$, J. Hart ${ }^{8}$, P.A.Hart ${ }^{9}$, M. Hauschild ${ }^{8}$, C.M.Hawkes ${ }^{8}$, E.Heflin ${ }^{4}$, R.J.Hemingway ${ }^{6}$, G. Herten ${ }^{10}$, R.D. Heuer ${ }^{8}$, J.C. Hill ${ }^{5}$, S.J. Hillier ${ }^{8}$, T. Hilse ${ }^{10}$, D.A. Hinshaw ${ }^{18}$, P.R. Hobson ${ }^{25}$, D. Hochman ${ }^{26}$, A. Höcker ${ }^{3}$, R.J.Homer' ${ }^{1}$, A.K. Honma ${ }^{28, a}$, R.E. Hughes-Jones ${ }^{16}$, R. Humbert $^{10}$, P. Igo-Kemenes ${ }^{11}$, H. Thssen ${ }^{11}$, D.C. Imrie ${ }^{25}$, A. Jawahery ${ }^{17}$, P.W. Jeffreys ${ }^{20}$, H. Jeremie ${ }^{18}$, M. Jimack ${ }^{1}$, M. Jones ${ }^{6}$, R.W.L. Jones ${ }^{8}$, P. Jovanovic ${ }^{1}$, C. Jui ${ }^{4}$, D. Karlen ${ }^{6}$, K. Kawagoe ${ }^{24}$, T. Kawamoto ${ }^{24}$, R.K. Keeler ${ }^{28}$, R.G. Kellogg ${ }^{17}$, B.W.Kennedy ${ }^{20}$, B. King ${ }^{8}$, J. King ${ }^{13}$, S. Kluth ${ }^{5}$, T. Kobayashi ${ }^{24}$, M. Kobel ${ }^{10}$, D.S. Koetke ${ }^{8}$, T.P. Kokott ${ }^{3}$, S. Komamiya ${ }^{24}$, R. Kowalewski ${ }^{8}$, R. Howard ${ }^{29}$, P. Krieger ${ }^{6}$, J. von Krogh ${ }^{11}$, P. Kyberd ${ }^{13}$, G.D. Lafferty ${ }^{16}$, H. Lafoux ${ }^{8}$, R. Lahmann ${ }^{17}$, J. Lauber ${ }^{8}$, J.G. Layter ${ }^{4}$, P.Leblanc ${ }^{18}$, P. Le Du ${ }^{21}$, A.M.Lee ${ }^{31}$, E.Lefebvre ${ }^{18}$, M.H.Lehto ${ }^{15}$, D. Lellouch ${ }^{26}$, C. Leroy ${ }^{18}$, J.Letts ${ }^{4}$, L.Levinson ${ }^{26}$, Z.Li' ${ }^{12}$, F.Liu ${ }^{29}$, S.L.Lloyd ${ }^{13}$, F.K.Loebinger ${ }^{16}$, G.D.Long ${ }^{17}$, B.Lorazo ${ }^{18}$, M.J. Losty ${ }^{7}$, X.C.Lou ${ }^{8}$, J. Ludwig ${ }^{10}$, A. Luig ${ }^{10}$, M. Mannelli ${ }^{8}$, S. Marcellini ${ }^{2}$, C. Markus ${ }^{3}$, A.J.Martin ${ }^{13}$, J.P. Martin ${ }^{18}$, T. Mashimo ${ }^{24}$, P.Mättig ${ }^{3}$, U.Maur ${ }^{3}$, J.McKenna ${ }^{29}$, T.J.McMahon ${ }^{1}$, A.I.McNab ${ }^{13}$, J.R.McNutt ${ }^{25}$, F.Meijers ${ }^{8}$, F.S.Merritt ${ }^{9}$, H. Mes ${ }^{7}$, A. Michelini ${ }^{8}$, R.P.Middleton ${ }^{20}$, G. Mikenberg ${ }^{26}$, J. Mildenberger ${ }^{6}$, D.J.Miller ${ }^{15}$, R. Mir $^{26}$, W. Mohr ${ }^{10}$, C.Moisan ${ }^{18}$, A. Montanari ${ }^{2}$, T.Mori ${ }^{24}$, M. Morii ${ }^{24}$, U. Müller ${ }^{3}$, B. Nellen ${ }^{3}$, H. Nguyen ${ }^{9, c}$, B. Nijjhar ${ }^{16}$, S.W. O'Neale ${ }^{1}$, F.G. Oakham ${ }^{7}$, F. Odorici ${ }^{2}$, H.O. Ogren ${ }^{12}$, C.J. Oram ${ }^{28, a}$, M.J. Oreglia ${ }^{9}$, S. Orito ${ }^{24}$, J.P.Pansart ${ }^{21}$, G.N. Patrick ${ }^{20}$, M.J. Pearce ${ }^{1}$, P.Pfister ${ }^{10}$, P.D.Phillips ${ }^{16}$, J.E. Pilcher ${ }^{9}$, J. Pinfold ${ }^{30}$, D. Pitman ${ }^{28}$, D.E. Plane ${ }^{8}$, P. Poffenberger ${ }^{28}$, B. Poli ${ }^{2}$, A. Posthaus ${ }^{3}$, T.W. Pritchard ${ }^{13}$, H. Przysiezniak ${ }^{18}$, M.W. Redmond ${ }^{8}$, D.L. Rees ${ }^{8}$, D. Rigby ${ }^{1}$, M. Rison ${ }^{5}$, S.A. Robins ${ }^{13}$, D. Robinson ${ }^{5}$, J.M. Roney ${ }^{28}$, E. Ros ${ }^{8}$, S. Rossberg ${ }^{10}$, A.M. Rossi ${ }^{2}$, M. Rosvick ${ }^{28}$, P. Routenburg ${ }^{30}$, Y. Rozen ${ }^{8}$, K. Runge $^{10}$, O. Runolfsson ${ }^{8}$, D.R. Rust ${ }^{12}$, M.Sasaki ${ }^{24}$, C. Sbarra ${ }^{2}$, A.D. Schaile ${ }^{8}$, O.Schaile ${ }^{10}$, F. Scharf ${ }^{3}$, P. Scharff-Hansen ${ }^{8}$, P.Schenk ${ }^{4}$, B.Schmitt ${ }^{3}$, H. von der Schmitt $^{11}$, M. Schröder ${ }^{12}$, H.C. Schultz-Coulon ${ }^{10}$, P.Schütz ${ }^{3}$, M. Schulz ${ }^{8}$, C. Schwick ${ }^{27}$, J. Schwiening ${ }^{3}$, W.G. Scott ${ }^{20}$, M.Settles ${ }^{12}$, T.G. Shears ${ }^{5}$, B.C. Shen ${ }^{4}$, C.H.Shepherd-Themistocleous ${ }^{7}$, P.Sherwood ${ }^{15}$, G.P. Siroli ${ }^{2}$, A. Skillman ${ }^{16}$, A. Skuja ${ }^{17}$, A.M. Smith ${ }^{8}$, T.J. Smith ${ }^{28}$, G.A.Snow ${ }^{17}$, R. Sobie ${ }^{28}$, R.W. Springer ${ }^{17}$, M. Sproston ${ }^{20}$, A. Stahl ${ }^{3}$, C. Stegmann ${ }^{10}$, K. Stephens ${ }^{16}$, J. Steuerer ${ }^{28}$, B. Stockhausen ${ }^{3}$, R. Ströhmer ${ }^{11}$, D. Strom ${ }^{19}$, P. Szymanski ${ }^{20}$, H. Takeda ${ }^{24}$, T. Takeshita ${ }^{24}$, S. Tarem ${ }^{26}$, M. Tecchio ${ }^{9}$, P. Teixeira-Dias ${ }^{11}$, N. Tesch ${ }^{3}$, M.A. Thomson ${ }^{15}$, E. Torrente ${ }^{22, d}$, S. Towers ${ }^{6}$, T. Tsukamoto ${ }^{24}$, M.F. Turner-Watson ${ }^{8}$, D. Van den plas $^{18}$, R. Van Kooten ${ }^{12}$, G. Vasseur ${ }^{21}$, M. Vincter ${ }^{28}$, A. Wagner ${ }^{27}$, D.L. Wagner ${ }^{9}$, C.P. Ward ${ }^{5}$, D.R. Ward ${ }^{5}$, J.J. Ward ${ }^{15}$, P.M. Watkins ${ }^{1}$, A.T. Watson ${ }^{1}$, N.K. Watson ${ }^{7}$, P. Weber ${ }^{6}$, P.S. Wells ${ }^{8}$, N. Wermes ${ }^{3}$, B. Wilkens ${ }^{10}$, G.W.Wilson ${ }^{4}$, J.A. Wilson ${ }^{1}$, V-H. Winterer ${ }^{10}$, T. Wlodek ${ }^{26}$, G. Wolf ${ }^{26}$, S. Wotton ${ }^{11}$, T.R. Wyatt ${ }^{16}$, A. Yeaman $^{13}$, G. Yekutieli ${ }^{26}$, M. Yurko ${ }^{18}$, W. Zeuner ${ }^{8}$, G.T.Zorn ${ }^{17}$

\footnotetext{
'School of Physics and Space Research, University of Birmingham, Birmingham B15 2TT, UK

${ }^{2}$ Dipartimento di Fisica dell' Università di Bologna and INFN, I-40126 Bologna, Italy

${ }^{3}$ Physikalisches Institut, Universität Bonn, D-53115 Bonn, Germany

${ }^{4}$ Department of Physics, University of California, Riverside CA 92521, USA

${ }^{5}$ Cavendish Laboratory, Cambridge CB3 OHE, UK

${ }^{6}$ Carleton University, Department of Physics, Colonel By Drive, Ottawa, Ontario K1S 5B6, Canada

${ }^{7}$ Centre for Research in Particle Physics, Carleton University, Ottawa, Ontario K1S 5B6, Canada

${ }^{8} \mathrm{CERN}$, European Organisation for Particle Physics, CH-1211 Geneva 23, Switzerland

${ }^{9}$ Enrico Fermi Institute and Department of Physics, University of Chicago, Chicago IL 60637, USA

${ }^{10}$ Fakultät für Physik, Albert Ludwigs Universität, D-79104 Freiburg, Germany

${ }^{11}$ Physikalisches Institut, Universität Heidelberg, D-69120 Heidelberg, Germany

${ }^{12}$ Indiana University, Department of Physics, Swain Hall West 117, Bloomington IN 47405, USA
} 


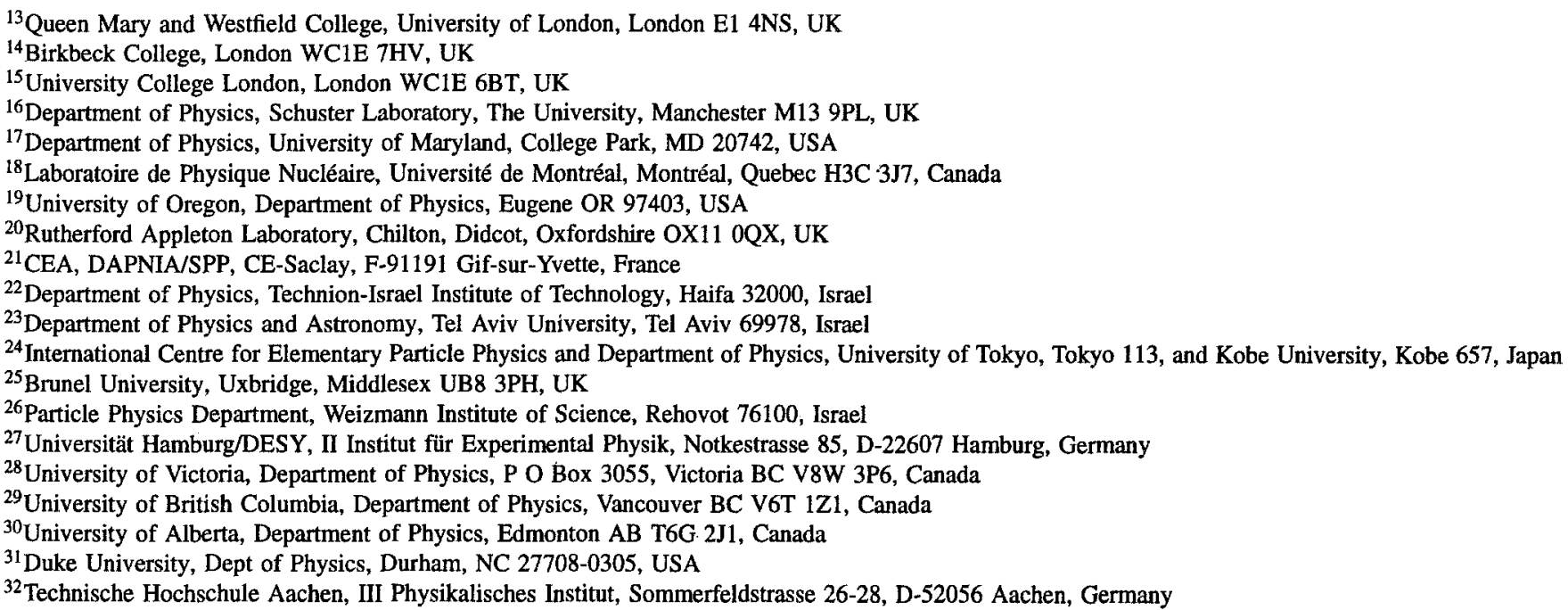

Received: 14 July 1994

\begin{abstract}
A search for the neutral Higgs bosons $\mathrm{h}^{0}$ and $\mathrm{A}^{0}$, predicted by the Minimal Supersymmetric Extension of the Standard Model (MSSM), has been performed by the OPAL Collaboration at LEP. The analysis was based on approximately $75 \mathrm{pb}^{-1}$ of data taken at centre-of-mass energies in the vicinity of the $Z^{0}$ resonance. No Higgs boson signals have been detected. Using, in addition, an upper limit on the contribution of non-Standard Model processes to the $Z^{0}$ boson width, almost the entire MSSM parameter space that can be reached at present LEP energies has been excluded. In particular, at the $95 \%$ confidence level, our results imply that $m_{\mathrm{h}^{0}}>44.5 \mathrm{GeV} / \mathrm{c}^{2}$ and $m_{\mathrm{A}^{0}}>24.3 \mathrm{GeV} / \mathrm{c}^{2}$, for $\tan \beta \geq 1$. The sensitivity to this assumption is discussed and the search results are also interpreted in the context of general two-doublet models. ${ }^{1}$
\end{abstract}

\section{Introduction}

Theories of the electroweak interaction with local gauge invariance introduce spontaneous symmetry breaking to allow for the gauge bosons $\mathrm{W}^{ \pm}$and $\mathrm{Z}^{0}$ to acquire mass while maintaining renormalizability. Such theories predict the existence of one or several scalar particles, the Higgs bosons [1]. To date, these particles have not been observed.

The Minimal Standard Model (SM) [2] is the simplest of these theories. It includes one doublet of complex Higgs fields to achieve symmetry breaking and predicts the existence of a single Higgs boson, $\mathrm{H}_{\mathrm{SM}}^{0}$, with unspecified mass but well defined couplings. The latter fix the cross section for Higgs boson production in association with a virtual $\mathrm{Z}^{0}$ boson, $\mathrm{Z}^{*}$, through the "bremsstrahlung" process, $\mathrm{e}^{+} \mathrm{e}^{-} \rightarrow \mathrm{Z}^{0} \rightarrow \mathrm{H}_{\mathrm{SM}}^{0} \mathrm{Z}^{*}[3]$ and determine the $\mathrm{H}_{\mathrm{SM}}^{0}$ decay modes, provided that the Higgs boson mass, $m_{\mathrm{H}_{\mathrm{SM}}^{0}}$, is specified.

\footnotetext{
1

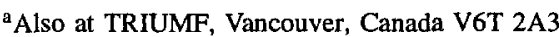

${ }^{b}$ Now at Texas Technical Institute, Lubbock, USA

${ }^{c}$ Now at Fermilab, P O Box 500, Batavia IL 60510, USA

${ }^{d}$ Now at University of Berne, CH-3012 Berne, Switzerland
}

Ever since the $\mathrm{e}^{+} \mathrm{e}^{-}$collider LEP at CERN came into operation, a considerable effort has been devoted to the search for the SM Higgs boson. The four LEP experiments, ALEPH, DELPHI, L3 and OPAL, have analysed up to 2 million hadronic $Z^{0}$ decays each, but no signal has been found. The current lower limits for $m_{\mathrm{H}_{S M}^{0}}$ are approximately $60 \mathrm{GeV} / \mathrm{c}^{2}$ from each experiment $[4,5]$. This limit cannot be significantly improved at the present LEP energy [6]; however, the searches will be continued at LEP 200, extending the sensitivity to $m_{\mathrm{H}_{\mathrm{SM}}^{0}} \approx 90 \mathrm{GeV} / \mathrm{c}^{2} \quad[7,8]$. Later these could be pursued at the hadron collider LHC [9] and perhaps at a future $\mathrm{e}^{+} \mathrm{e}^{-}$linear collider [10] covering eventually the mass range up to approximately $1 \mathrm{TeV} / \mathrm{c}^{2}$ which is the theoretical bound for $m_{\mathrm{H}_{\mathrm{SM}}^{\mathrm{0}}}$ based on vacuum stability and renormalizability [11].

Despite the success of the SM in describing elementary particle phenomena at the electroweak energy scale and below, the theory has considerable shortcomings. For example, it does not predict the mass spectrum and family structure of fermions. There is also the problem of quadratically divergent radiative corrections to the Higgs boson mass often referred to as the "naturalness problem". While in the SM these are cancelled by artificial fine-tuning of model parameters, supersymmetric (SUSY) models provide a more elegant solution: the divergent loops from standard particles are cancelled by equivalent loops of the SUSY partners [12].

The implementation of SUSY necessitates a second doublet of complex Higgs fields. One of the doublets, with vacuum expectation value (VEV) $v_{2}$, couples only to up-type fermions while the other, with vacuum expectation value $v_{1}$, couples only to down-type fermions. The VEV-ratio $\tan \beta=v_{2} / v_{1}$ is a free parameter of the model. While it is expected to be in the range $1 \leq \tan \beta \lessgtr m_{\mathrm{t}} / m_{\mathrm{b}} \approx 40\left(m_{\mathrm{t}}\right.$ and $m_{\mathbf{b}}$ are the top and bottom quark masses), values less than 1 cannot be excluded a priori [13].

The Minimal Supersymmetric Extension of the Standard Model, MSSM, predicts an extended Higgs sector with five physical Higgs bosons: one pair of charged particles, $\mathrm{H}^{ \pm}$, two CP-even neutral scalars and one CP-odd neutral particle, $\mathrm{A}^{0}$. The two CP-even scalar fields mix with an angle $\alpha$ 
to produce the two physical states $\mathrm{h}^{0}$ and $\mathrm{H}^{0}\left(m_{\mathrm{h}^{0}}<m_{\mathrm{H}^{0}}\right.$ by definition). At the tree level all Higgs boson masses and couplings can be expressed in terms of two independent parameters, for example $m_{\mathrm{h}^{0}}$ and $m_{\mathrm{A}^{0}}$, and the masses obey the following relations:

$m_{\mathrm{h}^{0}}<m_{\mathrm{Z}^{0}}<m_{\mathrm{H}^{0}}$,

$m_{\mathrm{h}^{0}}<m_{\mathrm{A}^{0}}<m_{\mathrm{H}^{0}}$

$m_{\mathrm{H}^{ \pm}}>m_{\mathrm{W}}$.

Of these tree level relations, the first has perhaps the greatest phenomenological impact since it limits the mass of the lightest Higgs boson, $\mathrm{h}^{0}$, from above, implying that a decisive test of the MSSM can be done, either at present accelerators or at those planned for the near future.

The above mass relations are modified when one-loop radiative corrections due to heavy quarks, in particular the top quark, are taken into account [14]. It is important to note, however, that even with radiative corrections included, the mass of the $\mathrm{h}^{0}$ boson is constrained to less than $\approx 140$ $\mathrm{GeV} / \mathrm{c}^{2}$ (if $\tan \beta \geq 1$ is assumed) ${ }^{1}$, as long as the top quark is not heavier than $200 \mathrm{GeV} / \mathrm{c}^{2}$, as suggested by the precise LEP measurements of the electroweak parameters $[16,17,18]$ and by the CDF experiment at Fermilab [19].

The MSSM Higgs bosons can be produced at LEP through the processes [12]

$\mathrm{Z}^{0} \rightarrow \mathrm{h}^{0} \mathrm{Z}^{*} \quad$ Higgs boson "bremsstrahlung"

$\mathrm{Z}^{0} \rightarrow \mathrm{h}^{0} \mathrm{~A}^{0} \quad$ Higgs boson "pair production".

The pair production process is kinematically restricted to $m_{\mathrm{h}^{0}}+m_{\mathrm{A}^{0}} \leq m_{\mathrm{Z}^{0}}$. Nevertheless, the "bremsstrahlung" process does not directly involve the $\mathrm{A}^{0}$ boson and can be used to probe regions of the MSSM parameter space with arbitrarily large $m_{\mathrm{A}^{0}}$. The cross sections are related to those of SM processes by

$\sigma\left(\mathrm{Z}^{0} \rightarrow \mathrm{h}^{0} \mathrm{Z}^{*}\right)=\sin ^{2}(\beta-\alpha) \cdot \sigma_{\mathrm{SM}}\left(\mathrm{Z}^{0} \rightarrow \mathrm{H}_{\mathrm{SM}}^{0} \mathrm{Z}^{*}\right)$

$$
\begin{aligned}
& \sigma\left(\mathrm{Z}^{0} \rightarrow \mathrm{h}^{0} \mathrm{~A}^{0}\right)=\cos ^{2}(\beta-\alpha) \\
& \quad \frac{1}{2} Q\left(1, m_{\mathrm{h}^{0}}^{2} / m_{\mathrm{Z}^{0}}^{2}, m_{\mathrm{A}^{0}}^{2} / m_{\mathrm{Z}^{0}}^{2}\right)^{3} \cdot \sigma_{\mathrm{SM}}\left(\mathrm{Z}^{0} \rightarrow \nu \bar{\nu}\right),
\end{aligned}
$$

where $Q(x, y, z)=\left[(x-y-z)^{2}-4 y z\right]^{1 / 2}$ is the usual twobody decay phase space factor and $\sigma_{\mathrm{SM}}\left(\mathrm{Z}^{0} \rightarrow \nu \bar{\nu}\right)$ is the cross section of the $Z^{0}$ decaying into neutrinos of any single family. The two processes are in a sense complementary to each other: in those parts of the $\left(m_{\mathrm{h}^{0}}, m_{\mathrm{A}^{0}}\right)$ parameter space where the first process is suppressed by a small value of $\sin ^{2}(\beta-\alpha)$, the second is enhanced by a large $\cos ^{2}(\beta-\alpha)$, and vice versa. Eq. (1) also implies that the searches for the SM process $\mathrm{Z}^{0} \rightarrow \mathrm{H}_{\mathrm{SM}}^{0} \mathrm{Z}^{*}$ can be interpreted in the MSSM context as searches for the process $\mathrm{Z}^{0} \rightarrow \mathrm{h}^{0} \mathrm{Z}^{*}$ with reduced sensitivity, provided that differences in the detection efficiencies, due to the different decay branching fractions of the $\mathrm{h}^{0}$ and $\mathrm{H}_{\mathrm{SM}}^{0}$ bosons, are taken into account.

The observable final states of the two processes are determined by the decay properties of the $\mathrm{h}^{0}$ and $\mathrm{A}^{0}$ bosons. The MSSM Higgs bosons, like the SM Higgs boson, couple

\footnotetext{
1 The upper limit of $m_{\mathrm{h}} 0$ may slightly vary if higher order corrections are added or if other perturbatively valid supersymmetric Higgs sectors are considered [15]
}

preferentially to the heaviest fermion pairs which are kinematically allowed. Additionally, in the MSSM the couplings also depend on the angles $\alpha$ and $\beta$. For $\tan \beta \geq 1$ and for masses greater than $2 m_{\mathrm{b}} \approx 10 \mathrm{GeV} / \mathrm{c}^{2}$, the dominant decay mode is into $b \bar{b}(\approx 90 \%)$ with $\approx 6-8 \%$ into $\tau^{+} \tau^{-}$[12]. In the region between $2 m_{\tau} \approx 3.5 \mathrm{GeV} / \mathrm{c}^{2}$ and $2 m_{\mathrm{b}}, \tau^{+} \tau^{-}$ and cé final states compete with each other. Between $2 m_{\mu}=$ $212 \mathrm{MeV} / \mathrm{c}^{2}$ and $2 m_{\tau}$ the MSSM predictions are not reliable due to large QCD corrections, and the searches must be decay-mode independent. For masses below $2 m_{\mu}$, only the channels $\mathrm{e}^{+} \mathrm{e}^{-}$and $\gamma \gamma$ (through heavy quark loops) are open; the searches have to deal with uncertainties in the relative rates and one has to allow for the possibility that the lifetime of a very light Higgs boson is sufficiently long for it to have a significant probability for escaping from the detector.

The domain $m_{\mathrm{A}^{0}}<m_{\mathrm{h}^{0}} / 2$, which becomes available by the inclusion of one-loop radiative corrections [20], deserves particular attention. There, the decay $\mathrm{h}^{0} \rightarrow \mathrm{A}^{0} \mathrm{~A}^{0}$ is kinematically possible and the great number of possible final states adds considerably to the complexity of the searches.

The results described in this work are based on $\mathrm{Z}^{0} \mathrm{de}-$ cays registered between 1990 and the end of 1993 and correspond to an integrated luminosity of approximately $75 \mathrm{pb}^{-1}$. Most of the data were collected at a centre of mass energy, $E_{c m}=m_{Z^{0}}$, and a smaller fraction at various energies within $\pm 3 \mathrm{GeV}$ of $m_{\mathrm{Z}^{0}}$. Practically all features of the OPAL detector have been exploited: precise tracking and calorimetric measurements, lepton identification and b-flavour tagging. The OPAL detector [21] and many of the techniques employed in this work have been described in previous publications which will be quoted in due course and to which the reader should refer for more information.

This paper is structured as follows: In Sect. 2 we describe the theoretical framework of the MSSM which includes calculations of one-loop radiative corrections, and the mathematical methods used to extract mass limits. In Sects. 3, 4 and 5 we assume $\tan \beta \geq 1$. In particular, in Sect. 3 we reassess the early, decay-mode independent, searches for the SM Higgs boson over the low mass range below $\approx 10$ $\mathrm{GeV} / \mathrm{c}^{2}[22,23]$, where the final states vary rapidly with mass. Used together with other constraints, these searches lead to the unambiguous exclusion of $h^{0}$ with mass in the range $0<m_{\mathrm{h}^{0}}<11.3 \mathrm{GeV} / \mathrm{c}^{2}$, regardless of $m_{\mathrm{A}^{0}}$. Sections 4 and 5 deal with the high mass domain, $m_{\mathrm{h}^{0}}>10$ $\mathrm{GeV} / \mathrm{c}^{2}$. We consider separately the region $m_{\mathrm{A}^{0}}>2 m_{\tau}$, where the MSSM provides firm predictions for the $A^{0}$ final states and the domain $m_{\mathrm{A}^{0}}<2 m_{\tau}$, where this is not the case. There again, the results rely in part on previously published searches which we quote without describing in detail; we rather concentrate on new analyses, unpublished so far. In Sect. 6 we summarise the results and discuss our mass limits within the strict MSSM framework. We also consider the case $\tan \beta<1$. The interpretation of the results in the context of more general models with two doublets of Higgs fields is presented in Sect. 7.

\section{Theoretical framework and analysis methods}

The present analysis is based on work quoted in Refs. [14, $20,24,25]$ and, in particular, on the relation [24]: 
$m_{\mathrm{h}^{0}}^{2}=\frac{1}{2}\left(m_{\mathrm{A}^{0}}^{2}+m_{\mathrm{Z}^{0}}^{2}+\epsilon-\Delta\right)$

with

$\Delta=\sqrt{\left(m_{\mathrm{A}^{0}}^{2}+m_{\mathrm{Z}^{0}}^{2}+\epsilon\right)^{2}-4 m_{\mathrm{A}^{0}}^{2} m_{Z^{0}}^{2} \cos ^{2} 2 \beta-4 \epsilon m_{\mathrm{A}^{0}}^{2} \sin ^{2} \beta-4 \epsilon m_{Z^{0}}^{2} \cos ^{2} \beta}$

The one-loop radiative corrections imply $\epsilon>0$, with

$\epsilon=\frac{3 \alpha_{W} m_{\mathrm{t}}^{4}}{2 \pi m_{\mathrm{W}}^{2} \sin ^{2} \beta} \log \left(\frac{m_{\mathrm{S}}^{2}}{m_{\mathrm{t}}^{2}}\right)$.

Here $m_{\mathrm{W}}$ is the $\mathrm{W}^{ \pm}$boson mass and $\alpha_{W}=\alpha / \sin ^{2} \theta_{W}$ the electroweak coupling constant. The SUSY partners of the quarks are all assumed to be degenerate with a common mass scale, $m_{\mathrm{S}}$. In the adopted approximation, only the loop correction due to the top quark is explicitly considered while effects from lighter quarks are not. The expression for $\epsilon$ shows a fourth power dependence on the top quark mass, $m_{\mathrm{t}}$, and a logarithmic dependence on $m_{\mathrm{S}}$. To date, the top quark mass is not known with precision. Based on results from the TEVATRON $[19,26]$ as well as on the interpretation of the precise LEP electroweak data within the context of the MSSM [18] we assume $m_{\mathrm{t}} \leq 200 \mathrm{GeV} / \mathrm{c}^{2}$ and, on general theoretical grounds, $m_{\mathrm{S}} \leq 1 \mathrm{TeV} / \mathrm{c}^{2}$ [12]. Consequently, $0 \leq \epsilon \leq \epsilon_{\max }$ where $\epsilon_{\max } \approx(75 / \sin \beta)^{2}$ $\left(\mathrm{GeV} / \mathrm{c}^{2}\right)^{2}$.

To project the MSSM parameter space on the $\left(m_{\mathrm{h}^{0}}, m_{\mathrm{A}^{0}}\right)$ plane we rewrite Eq. (3) in the form [27]

$\mathscr{B} y^{3}+\mathscr{B} y^{2}+\mathscr{C} y+\mathscr{D}=0$

with

$y=\sin ^{2} \beta$

$\mathscr{C}=4 m_{\mathrm{A}^{0}}^{2} m_{\mathrm{Z}^{0}}^{2}$

$\mathscr{B}=-\mathscr{B}$

$\mathscr{C}=m_{\mathrm{h}^{0}}^{4}-\left(m_{\mathrm{A}^{0}}^{2}+m_{\mathrm{Z}^{0}}^{2}\right) m_{\mathrm{h}^{0}}^{2}+\epsilon^{\prime}\left(m_{\mathrm{A}^{0}}^{2}-m_{\mathrm{Z}^{0}}^{2}\right)+m_{\mathrm{A}^{0}}^{2} m_{\mathrm{Z}^{0}}^{2}$

$\mathscr{D}=\epsilon^{\prime}\left(m_{\mathrm{Z}^{0}}^{2}-m_{\mathrm{h}^{0}}^{2}\right)$

$\epsilon^{\prime}=\epsilon \sin ^{2} \beta$.

The allowed parameter space is defined as the portion of the $\left(m_{\mathrm{h}^{0}}, m_{\mathrm{A}^{0}}\right)$ plane where the cubic equation (5) provides at least one solution with $0 \leq \sin ^{2} \beta \leq 1$; this domain is shown in Fig. 1, to the left of the full curve. The region to the left of the dashed line is the region allowed at the tree level. The inclusion of one-loop radiative corrections considerably increases the parameter space towards large $m_{\mathrm{h}^{0}}$ and into the region $m_{\mathrm{A}^{0}}<m_{\mathrm{h}^{0}}$, below the dashed line.

To determine whether a given point $\left(m_{\mathrm{h}^{0}}, m_{\mathrm{A}^{0}}\right)$ is experimentally excluded or not, we vary $\epsilon$ between zero and $\epsilon_{\max }$ and calculate in each case the MSSM parameters $\tan \beta$ and $\sin ^{2}(\beta-\alpha)$ which fix the Higgs boson production cross sections and branching ratios. If Eq. (5) yields several solutions, these are examined one by one. The number, $n_{i}$, of expected events in a search for a given final state $i$ with branching ratio $B r_{i}$ is

$n_{i}=\sigma \cdot B r_{i} \cdot \mathscr{L}_{i} \cdot \eta_{i}$

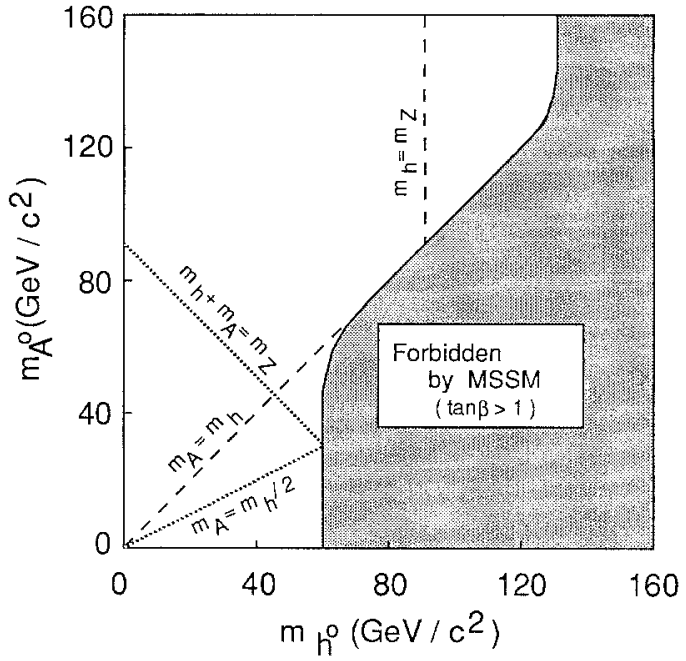

Fig. 1. The MSSM parameter space as defined in Ref. [14] for $\tan \beta \geq 1$ including the effect of one-loop radiative corrections. The shaded area is forbidden by the MSSM. The dashed line indicates the limits, $m_{\mathrm{A}^{0}}=m_{\mathrm{h}^{0}}$ and $m_{\mathrm{h}^{0}}=m_{\mathrm{Z}^{0}}$, which would be valid at the tree level. The kinematic limits for the processes $Z^{0} \rightarrow h^{0} A^{0}$ and $h^{0} \rightarrow A^{0} A^{0}$ are indicated by dotted lines

where $\mathscr{L}_{i}$ is the integrated luminosity of the data sample on which that search is based and $\eta_{i}$ the detection efficiency. The expressions for the production cross section, $\sigma$, and the Higgs boson decay branching ratios can be found for example in Ref. [12]. For $m_{\mathrm{A}^{0}}>2 m_{\mathrm{b}}$, the branching ratios of $h^{0}$ and $A^{0}$ into $b \bar{b}$ and $\tau^{+} \tau^{-}$final states are fixed in $a$ model-independent way by the ratio of the fermion masses. For $2 m_{\tau}<m_{\mathrm{A}^{0}}<2 m_{\mathrm{b}}$, the branching ratios into $c \overline{\mathrm{c}}$ and $\tau^{+} \tau^{-}$final states depend on $\alpha$ and $\beta$ which are given by the MSSM for each value of $\epsilon$ considered. In the region where the $\mathrm{h}^{0} \rightarrow \mathrm{A}^{0} \mathrm{~A}^{0}$ channel is open, its branching fraction is calculated using Ref. [25] which includes one-loop radiative corrections. The detection efficiency, $\eta_{i}$, includes the geometrical acceptance, the trigger efficiency and the effect of the selection cuts. It is obtained, as a function of $m_{\mathrm{h}^{0}}$ and $m_{\mathrm{A}^{0}}$, from Monte Carlo simulations of the Higgs boson signal. Typically, a grid of points is simulated, separated by 5 $\mathrm{GeV} / \mathrm{c}^{2}$ in $m_{\mathrm{h}^{0}}$ and $m_{\mathrm{A}^{0}}$, and efficiencies at intermediate masses are obtained by interpolation. In calculating the expected number of events, $n_{i}$, we conservatively reduced the detection efficiencies by their one standard deviation error.

The total number of expected events is obtained by summing over the $\mathbf{Z}^{0} \rightarrow \mathbf{h}^{0} \mathbf{Z}^{*}$ and $Z^{0} \rightarrow \mathbf{h}^{0} \mathrm{~A}^{0}$ final states $(i$ and $j)$. To decide if a given point, $\left(m_{\mathrm{h}^{0}}, m_{\mathrm{A}^{0}}\right)$, is excluded or not, one selects the smallest expectation for a Higgs boson signal,

$$
\begin{aligned}
& \mathscr{N}\left(m_{\mathrm{h}^{0}}, m_{\mathrm{A}^{0}}\right)=\min _{0 \leq \epsilon \leq \epsilon_{m a x}}\left[\sum_{i} n_{i}^{\mathrm{Z}^{0} \rightarrow \mathrm{h}^{0} \mathrm{Z}^{*}}\left(m_{\mathrm{h}^{0}}, m_{\mathrm{A}^{0}}, \epsilon\right)\right. \\
& \left.+\sum_{j} n_{j}^{\mathrm{Z}^{0} \rightarrow \mathrm{h}^{0} \mathrm{~A}^{0}}\left(m_{\mathrm{h}^{0}}, m_{\mathrm{A}^{0}}, \epsilon\right)\right],
\end{aligned}
$$

while varying the radiative correction parameter $\epsilon$. The point is excluded if $\mathscr{N}$ is larger than the experimental $95 \%$ confidence level (CL) upper limit obtained from the data.

In most of our searches Poisson statistics are applied to determine the $95 \% \mathrm{CL}$ upper limits, since close to the mass limits the number of expected Higgs boson events is 
small. In those cases where the background could be almost completely supressed, the few surviving data events were treated as Higgs boson candidates. The probability to observe $N$ events while the model predicts $\lambda$ events is given by the Poisson distribution function

$P_{\lambda}(N)=\frac{1}{N !} \mathrm{e}^{-\lambda} \lambda^{N}$

and the $95 \%$ CL upper limit is given by

$\mathrm{e}^{-\lambda} \sum_{n=0}^{N}\left(\frac{1}{n !} \lambda^{n}\right)=0.05$.

For $N=0$ or 1 , Eq. (9) gives $\lambda=3$ or 4.7 , respectively. In the present case, however, the observed candidate events have well measured Higgs boson masses, $m$; the 95\% CL upper limit for the signal may thus be modified in a way which takes into account the experimental mass resolution, $\Delta m$. We adopted a simple, conservative approach which is to increase the $95 \% \mathrm{CL}$ upper limit from 3 to 4.7 in the mass range $m \pm 2 \Delta m$ of a single isolated event.

In the cases where the background could not be fully suppressed it was determined by Monte Carlo simulation and subtracted from the data. If $N$ data events remain after the selection and the number of expected background events is $\mu_{B} \pm \sigma_{\mu_{B}}$, a conservative $95 \% \mathrm{CL}$ upper limit, $\lambda$, for a possible signal is obtained by solving the following equation $[28,29]$ :

$\mathrm{e}^{-\left(\mu_{B}+\lambda\right)} \sum_{n=0}^{N} \frac{\left(\mu_{B}+\lambda\right)^{n}}{n !}=0.05 \mathrm{e}^{-\mu_{B}} \sum_{n=0}^{N} \frac{\mu_{B}^{n}}{n !}$.

In order to take into account the uncertainty of the background estimate, we used $\mu_{B}-\sigma_{\mu_{B}}$ instead of $\mu_{B}$ in the above formula to determine $\lambda$.

\section{The low mass domain $m_{h^{0}}<10 \mathrm{GeV} / \mathrm{c}^{2}$}

Very light Higgs bosons, with mass well below $2 m_{\mu}$, have long lifetimes and may escape the detector. On the other hand, if the Higgs boson mass is in the range $2 m_{\mu}<$ $m_{\mathrm{h}^{0}, \mathrm{~A}^{0}}<2 m_{\tau}$, its decay modes are uncertain due to large QCD corrections. These problems arise equally for SM and MSSM Higgs bosons.

A search strategy was adopted which led to a decaymode independent exclusion of the SM Higgs boson from the entire range $0 \leq m_{\mathrm{H}_{\mathrm{SM}}^{0}}<11.3 \mathrm{GeV} / \mathrm{c}^{2} \quad(95 \% \mathrm{CL})$ $[22,23]$. The search for the process $\mathrm{Z}^{0} \rightarrow \mathrm{H}_{\mathrm{SM}}^{0} \mathrm{Z}^{*}$ covers the quoted mass range in two steps:

For a very low mass Higgs boson, with $m_{\mathrm{H}_{\mathrm{SM}}^{0}}<2 m_{\mu}$, the two decay channels $\mathrm{H}_{\mathrm{SM}}^{0} \rightarrow \mathrm{e}^{+} \mathrm{e}^{-}$and $\mathrm{H}_{\mathrm{SM}}^{0} \rightarrow \gamma \gamma$ were considered [22]. The Higgs boson lifetime, a function of $m_{\mathrm{H}_{\mathrm{SM}}^{0}}$, was taken into account in the determination of the detection efficiencies. Two final states of the $Z^{*}$ were used in a complementary fashion: (a) the neutrino channel $\left(\mathrm{Z}^{*} \rightarrow \nu \bar{\nu}\right)$, for short-lived Higgs bosons which deposit their energy in the electromagnetic calorimeter and (b) the leptonic channel $\left(\mathrm{Z}^{*} \rightarrow \mathrm{e}^{+} \mathrm{e}^{-}, \mu^{+} \mu^{-}\right)$, for long-lived and thus invisible Higgs bosons, giving rise to acolinear pairs of energetic leptons. (The acolinearity angle is the complement of the 3 -dimensional angle between the two momentum vectors.)

For $2 m_{\mu}<m_{\mathrm{H}_{\mathrm{SM}}^{0}}<2 m_{\tau}$, the problem arising from the uncertainty on the Higgs boson decay modes was avoided by using the $\mathrm{Z}^{*} \rightarrow \mathrm{e}^{+} \mathrm{e}^{-}, \mu^{+} \mu^{-}$channels and searching for an acoplanar pair of inclusive leptons. (The acoplanarity angle is the complement of the angle between the projections of the two momentum vectors on the plane perpendicular to the beam direction.) The main background arises from radiative dilepton processes. Therefore, event topologies consistent with $\mathrm{Z}^{0} \rightarrow \ell^{+} \ell^{-} \gamma$ or $\mathrm{Z}^{0} \rightarrow \ell^{+} \ell^{-} \gamma \gamma$ (where the photons may have converted) were rejected. Since the removal of this background may have eliminated Higgs bosons decaying into purely electromagnetic final states, $\left(\mathrm{e}^{+} \mathrm{e}^{-}, \gamma \gamma, \pi^{0} \pi^{0}\right.$, etc...) such final states were explicitly searched for in the neutrino channel, $\mathrm{Z}^{*} \rightarrow \nu \bar{\nu}$, by looking for isolated energetic clusters in the electromagnetic calorimeter.

Interpreted in the MSSM context as a search for the process $\mathrm{Z}^{0} \rightarrow \mathrm{h}^{0} \mathrm{Z}^{*}$, the absence of a signal in this search yields an upper limit for $\sin ^{2}(\beta-\alpha)$ using Eq. (1). This limit, as a function of $m_{\mathrm{h}^{0}}$, is shown in Fig. 5 (a) of Ref. [23].

An independent constraint, on $\cos ^{2}(\beta-\alpha)$, is obtained from the precise LEP measurements of the $Z^{0}$ decay width, $\Gamma_{\mathrm{Z}^{0}}$. Compared to the $\mathrm{SM}$ prediction it yields an experimental upper limit of $\Gamma_{\mathrm{X}}<13.9 \mathrm{MeV}$ (95\% CL) [30] for the partial width of any non-standard decay, $Z^{0} \rightarrow X$. Assigning to " $\mathrm{X}$ " the MSSM channel $\mathrm{h}^{0} \mathrm{~A}^{0}$, the limit on $\Gamma_{\mathrm{X}}$ is converted, at every point of the $\left(m_{\mathrm{h}^{0}}, m_{\mathrm{A}^{0}}\right)$ plane, into a limit on $\cos ^{2}(\beta-\alpha)$ using Eq. (2). The comparison of these experimental limits on $\sin ^{2}(\beta-\alpha)$ and $\cos ^{2}(\beta-\alpha)$ with the prediction of the MSSM leads to the unambiguous exclusion of the entire domain $m_{\mathrm{h}^{0}}<11.3 \mathrm{GeV} / \mathrm{c}^{2} \quad(95 \% \mathrm{CL})$, for any value of $m_{\mathrm{A}^{0}}$. This exclusion is shown by the vertical dashed line in Fig. 2. The line labeled A indicates the exclusion one would obtain without the constraint on $\cos ^{2}(\beta-\alpha)$ from $\Gamma_{Z^{0}}$. One should note that the limit for $m_{\mathrm{h}^{0}}$ is determined mainly by the constraint on $\sin ^{2}(\beta-\alpha)$ and is rather insensitive to the precise value of $\Gamma_{\mathrm{X}}$.

\section{The high mass domain $m_{h^{0}}>10 \mathrm{GeV} / \mathrm{c}^{2}$}

For masses larger than $2 m_{\tau}$, the $\mathrm{h}^{0}$ and $\mathrm{A}^{0}$ boson decay branching ratios are well predicted by the MSSM. In the domain with $m_{\mathrm{h}^{0}}>10 \mathrm{GeV} / \mathrm{c}^{2}$ there remains, however, a narrow band, with $m_{\mathrm{A}^{0}}<2 m_{\tau}$, where our previous considerations regarding long lifetimes and uncertain final state predictions are still relevant for the $\mathrm{A}^{0}$ boson. We postpone the discussion of that band to the next section.

In the domain given by $m_{\mathrm{h}^{0}}>10 \mathrm{GeV} / \mathrm{c}^{2}$ and $m_{\mathrm{A}^{0}}>2 m_{\tau}$, we make use of the SM Higgs boson searches which were designed for $m_{\mathrm{H}_{\mathrm{SM}}^{0}}>2 m_{\mathrm{b}}$. Using Eq. (1), these searches are converted into limits on $\sin ^{2}(\beta-\alpha)$, after correcting for differences in the detection efficiencies of the two processes $Z^{0} \rightarrow H_{S M}^{0} Z^{*}$ and $Z^{0} \rightarrow h^{0} Z^{*}$ due to different decay branching ratios. Usually, these differences are small, except for $m_{\mathrm{A}^{0}}<m_{\mathrm{h}^{0}} / 2$ (below the dotted line in Fig. 1), where the decay $\mathrm{h}^{0} \rightarrow \mathrm{A}^{0} \mathrm{~A}^{0}$ is allowed. The limits on $\sin ^{2}(\beta-\alpha)$ are used in conjunction with limits on 


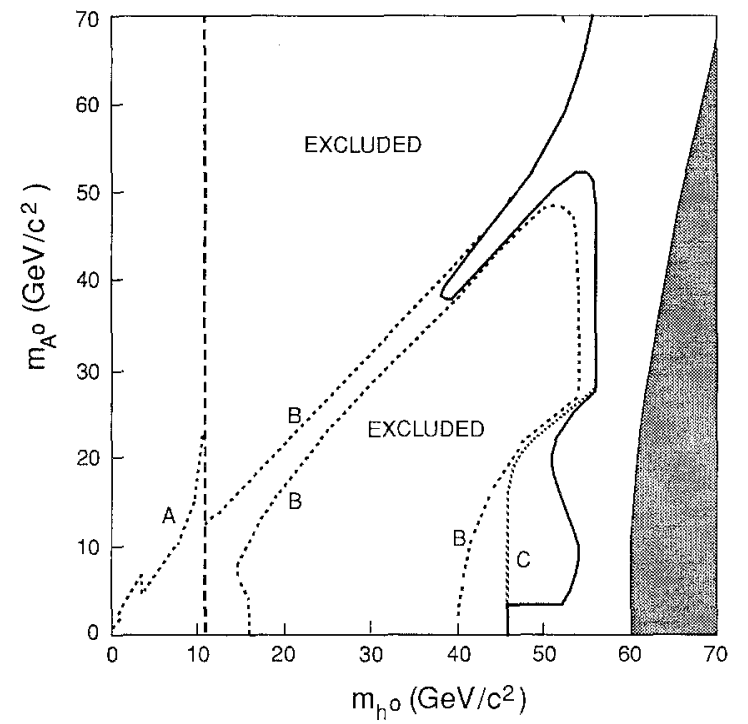

Fig. 2. Exclusions (95\% CL) obtained for $\tan \beta>1$ from searches for the SM Higgs boson. Curve A: low-mass searches [22, 23]. Vertical dashed line: low-mass searches in conjunction with the constraint from $\Gamma_{Z^{0}}$. Curves B: exclusion obtained from the high mass search [5]. Curve C: low- and high-mass searches in conjunction with the constraint from $\Gamma_{Z^{0}}$. Solid line: exclusion obtained by extending the high-mass search [5] also th the channels $Z^{0} \rightarrow h^{0} Z^{*} \rightarrow\left(q \bar{q} q \bar{q}, \tau^{+} \tau^{-} q \bar{q}, \tau^{+} \tau^{-} \tau^{+} \tau^{-}\right) \nu \bar{\nu}$

$\cos ^{2}(\beta-\alpha)$ which are obtained either from $\Gamma_{Z^{0}}$ (cf. discussion in the previous section) or from direct searches for $Z^{0} \rightarrow h^{0} A^{0}$ final states, using Eq. (2).

\subsection{Limits from the searches for the SM Higgs boson}

The most recent OPAL publication on searches for the SM Higgs boson [5] is based on data collected from 1990 to the end of 1993 , with an integrated luminosity of $\approx 75 \mathrm{pb}^{-1}$. The search comprises two final states of the $\mathrm{Z}^{0} \rightarrow \mathrm{H}_{\mathrm{SM}}^{0} \mathrm{Z}^{*}$ process: the neutrino channel $\left(\mathrm{Z}^{*} \rightarrow \nu \bar{\nu}\right)$ and the leptonic channels $\left(\mathrm{Z}^{*} \rightarrow \mathrm{e}^{+} \mathrm{e}^{-}, \mu^{+} \mu^{-}\right)$. In the first case one looks for an event topology with two acoplanar jets and missing energy, while in the second case for pairs of isolated, highmomentum, oppositely charged electrons or muons produced in association with hadronic jets. For a detailed description of the methods, the selection cuts and the results the reader is referred to Ref. [5].

The search resulted in two candidate events, one in the $\mathrm{Z}^{*} \rightarrow \nu \bar{\nu}$ channel, compatible with a Higgs boson mass of $25.1 \pm 3.0 \mathrm{GeV} / \mathrm{c}^{2}$, and one in the $\mathrm{Z}^{*} \rightarrow \mu^{+} \mu^{-}$channel, compatible with $m_{\mathrm{H}_{\mathrm{sM}}^{0}}=61.2 \pm 1.0 \mathrm{GeV} / \mathrm{c}^{2}$. These events are treated as Higgs boson candidates.

For the present purpose, the determination of the detection efficiencies in the $\mathrm{Z}^{*} \rightarrow \nu \bar{\nu}$ channel has been extended to cover the mass range from $10 \mathrm{GeV} / \mathrm{c}^{2}$ to $70 \mathrm{GeV} / \mathrm{c}^{2}$. In Fig. 2 the dashed lines labeled B indicate the 95\% CL exclusion which is obtained when the above search is interpreted in the MSSM context, including for the moment only $\mathrm{h}^{0} \rightarrow$ ff final states (i.e., setting the $\mathrm{h}^{0} \rightarrow \mathrm{A}^{0} \mathrm{~A}^{0}$ detection efficiencies to zero) in the calculation of the expected number of signal events. The two disconnected excluded regions, one above and one below the $m_{\mathrm{A}^{0}}=m_{\mathrm{h}^{0}}$ diagonal line, result from the fact that $\sin ^{2}(\beta-\alpha)$ is predicted to be zero on the diagonal. The sharp edge at $m_{\mathrm{h}^{0}}=54 \mathrm{GeV} / \mathrm{c}^{2}$ and $m_{\mathrm{A}^{0}}=27 \mathrm{GeV} / \mathrm{c}^{2}$ is due to the onset of the $\mathrm{h}^{0} \rightarrow \mathrm{A}^{0} \mathrm{~A}^{0}$ decay channel.

In Fig. 2 , the solid line (for $m_{\mathrm{A}^{0}}>27 \mathrm{GeV} / \mathrm{c}^{2}$ ) continued by the curve labeled $\mathrm{C}$ (for $m_{\mathrm{A}^{0}}<27 \mathrm{GeV} / \mathrm{c}^{2}$ ), shows the limit which is obtained when the constraint from $\Gamma_{\mathrm{Z}^{0}}$ is added. Since the $\mathrm{h}^{0} \rightarrow \mathrm{A}^{0} \mathrm{~A}^{0}$ decays are not used, this exclusion (which reaches $m_{\mathrm{h}^{0}}=46 \mathrm{GeV} / \mathrm{c}^{2}$ for low $m_{\mathrm{A}^{0}}$ and $m_{\mathrm{h}^{0}}=38 \mathrm{GeV} / \mathrm{c}^{2}$ for any $\left.m_{\mathrm{A}^{0}}\right)$ is free from any assumption concerning the $\mathrm{A}^{0}$ boson decay mode and valid down to $m_{\mathrm{A}^{0}}=0$.

The selection criteria of the search in the $\mathrm{Z}^{*} \rightarrow \nu \bar{\nu}$ channel were also applied to the processes $Z^{0} \rightarrow Z^{*} h^{0} \rightarrow$ $\nu \bar{\nu} \mathrm{A}^{0} \mathrm{~A}^{0} \rightarrow \nu \bar{\nu}\left(\mathrm{q} \bar{q} q \bar{q}, \tau^{+} \tau^{-} \mathrm{q} \bar{q}, \tau^{+} \tau^{-} \tau^{+} \tau^{-}\right)$. The detection efficiencies were typically smaller than those of the $\nu \bar{\nu} \mathrm{q} \bar{q}$ final states; for example, at $m_{\mathrm{h}^{0}}=50 \mathrm{GeV} / \mathrm{c}^{2}$ the $\nu \bar{\nu} \mathrm{q} \bar{q}$ efficiency was $45 \%$ [5] while the $\nu \bar{\nu} \mathrm{q} \overline{\mathrm{q}} \mathrm{q} \overline{\mathrm{q}}$ efficiencies were $47 \%, 30 \%$ and $18 \%$ for $m_{\mathrm{A}^{0}}=5,15$ and $25 \mathrm{GeV} / \mathrm{c}^{2}$, respectively. The inclusion of these channels extends the exclusion in the domain $2 m_{\tau}<m_{\mathrm{A}^{0}}<m_{\mathrm{h}^{0}} / 2$, from curve $\mathrm{C}$ to the solid line (Fig. 2).

\subsection{Dedicated searches for specific $\mathrm{Z}^{0} \rightarrow \mathrm{h}^{0} \mathrm{~A}^{0}$ final states}

The exclusion shown in Fig. 2 can be improved both in the region $m_{\mathrm{A}^{0}} \approx m_{\mathrm{h}^{0}}$ and at large $m_{\mathrm{h}^{0}}$ by searches for specific $Z^{0} \rightarrow h^{0} A^{0}$ final states. The cross section for this process, Eq. (2), is mainly limited by the phase space factor, $Q$, which drops to zero at the kinematic limit, $m_{\mathrm{h}^{0}}+m_{\mathrm{A}^{0}}=m_{\mathrm{Z}^{0}}$.

4.2.1 Final states with inclusive $\tau^{+} \tau^{-}$pairs. In the region $m_{\mathrm{A}^{0}} \approx m_{\mathrm{h}^{0}}$ the exclusion is improved by a search for the process $\mathrm{Z}^{0} \rightarrow \mathrm{h}^{0} \mathrm{~A}^{0} \rightarrow \tau^{+} \tau^{-} \mathrm{q} \overline{\mathrm{q}}$ where the $\tau^{+} \tau^{-}$ pair originates either from the $h^{0}$ or from the $A^{0}$ boson. For $m_{\mathrm{h}^{0}}>2 m_{\tau}$ and $m_{\mathrm{A}^{0}}>m_{\mathrm{h}^{0}} / 2$, the MSSM predicts branching ratios for $\mathrm{h}^{0} \rightarrow \tau^{+} \tau^{-}$and $\mathrm{A}^{0} \rightarrow \tau^{+} \tau^{-}$in the range 6-8\%, leading to comfortable event rates in these channels. Furthermore, the two isolated $\tau$ leptons provide a clean signal, well separated from the background.

The search for events with $\tau^{+} \tau^{-}$pairs is described in a previous publication [31]. The main signature was that of two oppositely charged, isolated, high-momentum tracks from one-prong decays of the $\tau$ leptons, with invariant mass greater than $10 \mathrm{GeV} / \mathrm{c}^{2}$. Although the conclusions of that publication with regards to mass limits need modifying in the light of radiative corrections, the methods remain valid. The signal detection efficiency of that search at, for example, $m_{\mathrm{h}^{0}}=45 \mathrm{GeV} / \mathrm{c}^{2}$ and $m_{\mathrm{A}^{0}}=45 \mathrm{GeV} / \mathrm{c}^{2}$ is $6.5 \%$.

This search has been applied to an event sample of $\approx$ $47 \mathrm{pb}^{-1}$ integrated luminosity. A few events, with hadronic mass smaller than $15 \mathrm{GeV} / \mathrm{c}^{2}$, survived the selection. These are compatible with background from higher-order "fourfermion" final states [32]. Due to the low hadronic mass, these events have no impact in the relevant region, $m_{\mathrm{A}^{0}} \approx$ $m_{\mathrm{h}^{0}}>30 \mathrm{GeV} / \mathrm{c}^{2}$. By adding this channel to the ones previously described, the $95 \% \mathrm{CL}$ exclusion in the region $m_{\mathrm{A}^{0}} \approx m_{\mathrm{h}^{0}}$ is improved: one obtains $m_{\mathrm{A}^{0}}>44.5 \mathrm{GeV} / \mathrm{c}^{2}$. 


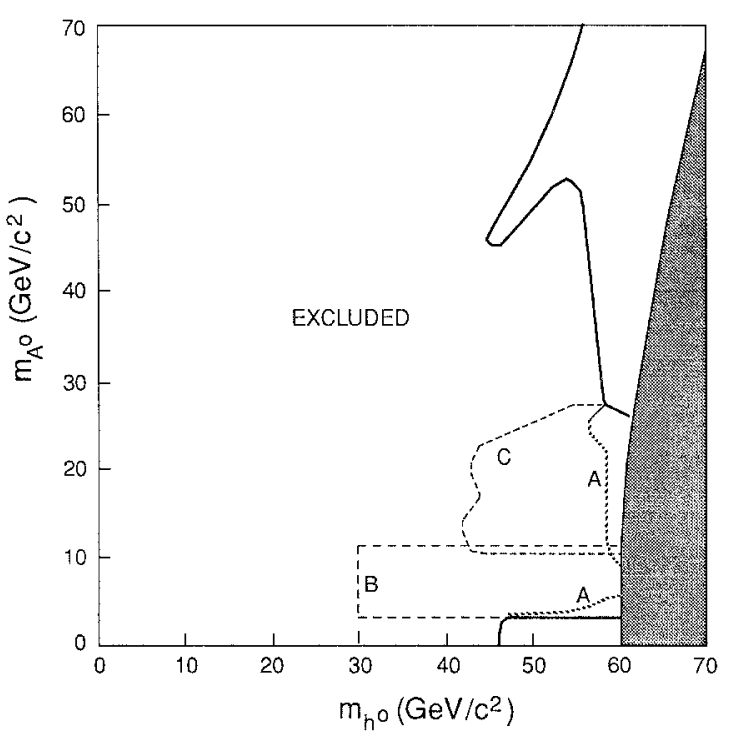

Fig. 3. Exclusion (95\% CL) obtained for $\tan \beta \geq 1$ by adding the results from the searches for $Z^{0} \rightarrow h^{0} A^{0}$ final states. Line $A$ : exclusion obtained by adding final states with inclusive $\tau^{+} \tau^{-}$pairs; line $\mathrm{B}$ : region where the $3\left(\tau^{+} \tau^{-}\right)$final state contributes; line $\mathrm{C}$ : region where the 3(b) final state contributes. Solid lines: global exclusion

This limit cannot be significantly improved at LEP I since it is close to the kinematic limit ${ }^{2}$.

The same search has also been applied to the process $Z^{0} \rightarrow h^{0} A^{0} \rightarrow\left(A^{0} A^{0}\right) A^{0}$ with either one or two $A^{0}$ bosons decaying into $\tau^{+} \tau^{-}$pairs. These channels are relevant for $2 m_{\tau}<m_{\mathrm{A}^{0}}<m_{\mathrm{h}^{0}} / 2$ and abundant for $m_{\mathrm{A}^{0}}<2 m_{\mathrm{b}}$. The simulation of the detection efficiencies has taken into account that the $\tau^{+} \tau^{-}$pairs could arise from the primary $\mathrm{A}^{0}$ boson or from cascade $\left(\mathrm{h}^{0} \rightarrow \mathrm{A}^{0} \mathrm{~A}^{0}\right) \mathrm{A}^{0}$ bosons. For the $\tau^{+} \tau^{-} q \bar{q} q \bar{q}$ final state the detection efficiencies were found to be similar to those of $\tau^{+} \tau^{-} \mathrm{q} \overline{\mathrm{q}}$ while for $\tau^{+} \tau^{-} \tau^{+} \tau^{-} \mathrm{q} \overline{\mathrm{q}}$ they were higher. The inclusion of these final states improves the limits in the region $2 m_{\tau}<m_{\mathrm{A}^{0}}<m_{\mathrm{h}^{0}} / 2$, extending them towards higher values of $m_{\mathrm{h}^{0}}$, as indicated by the line labeled A in Fig. 3.

4.2.2 The $3\left(\tau^{+} \tau^{-}\right)$final state. The region $2 m_{\tau}<m_{\mathrm{A}^{0}}<$ $2 m_{\mathrm{b}}$ is not fully excluded by the previous search for events with $\tau$ lepton pairs. Nevertheless, the process $\mathrm{Z}^{0} \rightarrow \mathrm{h}^{0} \mathrm{~A}^{0} \rightarrow$ $\left(\mathrm{A}^{0} \mathrm{~A}^{0}\right) \mathrm{A}^{0} \rightarrow 3\left(\tau^{+} \tau^{-}\right)$provides a clean signature with a relatively large cross section since in this mass range the $\mathrm{A}^{0} \rightarrow \tau^{+} \tau^{-}$channel is competing only with the $\mathrm{A}^{0} \rightarrow \mathrm{c} \overline{\mathrm{c}}$ final state. A specific search was carried out, concentrating on $m_{\mathrm{h}^{0}}>30 \mathrm{GeV} / \mathrm{c}^{2}$. The Higgs boson signal is expected to have a low charged particle multiplicity and several energetic, isolated leptons from the $\tau^{ \pm}$decays. In addition, there should be a sizeable missing energy due to the $\tau$-neutrinos and, for large $m_{\mathrm{h}^{0}}$, the event should also have high sphericity.

After applying the standard quality requirements for charged tracks and electromagnetic clusters (the precise se-

\footnotetext{
${ }^{2}$ An almost identical limit was obtained from a search for the $Z^{0} \rightarrow$ $h^{0} A^{0} \rightarrow q \bar{q} q \bar{q}$ (four-jet) final state, also described in Ref. [31]. Although the four-jet topology is more abundant than the $\tau^{+} \tau^{-} q \bar{q}$ final state, it suffers from QCD background which cannot be suppressed completely
}

lection is described in [33]), one required at least two charged tracks with momentum larger than $2 \mathrm{GeV} / \mathrm{c}$, polar angle between 45 and 135 degrees, opposite electric charge, and well isolated (isolation cone with a half-angle of 30 degrees) from any other charged track. The background, mainly from hadronic $Z^{0}$ decays, was completely suppressed by the following simple selection criteria:

Charged track multiplicity $<13$;

Total visible energy $<50 \mathrm{GeV}$;

Sphericity $>0.05$;

The event hemisphere containing the two isolated tracks and defined by the plane perpendicular to the vector sum of the two track momenta should not contain more than $0.5 \mathrm{GeV}$ in addition to the energy of the two tracks.

Applying this selection to a data sample of $\approx 47 \mathrm{pb}^{-1}$ integrated luminosity leaves no surviving candidate events. In the mass range relevant to this search, $m_{\mathrm{h}^{0}}>30 \mathrm{GeV} / \mathrm{c}^{2}$ and $4<m_{\mathrm{A}^{0}}<12 \mathrm{GeV} / \mathrm{c}^{2}$, the detection efficiencies vary between $2 \%$ (high $m_{\mathrm{A}^{0}}$ ) and $11 \%$ (low $m_{\mathrm{A}^{0}}$ ). The region where this analysis has an impact on the exclusion is indicated by the curve labeled B in Fig. 3.

4.2.3 The 3(b $\bar{b})$ final state. In this section we describe a search for the decay $\mathrm{Z}^{0} \rightarrow \mathrm{h}^{0} \mathrm{~A}^{0} \rightarrow\left(\mathrm{A}^{0} \mathrm{~A}^{0}\right) \mathrm{A}^{0} \rightarrow 3(\mathrm{~b} \overline{\mathrm{b}})$ which improves the sensitivity in the region $m_{\mathrm{h}^{0}}>40$ $\mathrm{GeV} / \mathrm{c}^{2}, 2 m_{\mathrm{b}}<m_{\mathrm{A}^{0}}<m_{\mathrm{h}^{0}} / 2$, not fully excluded by the previous searches. Since in this region the dominant decay of the $\mathrm{A}^{0}$ boson is $\mathrm{A}^{0} \rightarrow \mathrm{b} \overline{\mathrm{b}}$ with a branching ratio larger than $90 \%$, this final state provides a copious Higgs boson signal with many b-flavoured hadrons. To reduce the QCD background to a tolerable level, kinematic cuts were applied and b-flavoured hadrons were tagged via displaced secondary vertices. To achieve the best possible b-tagging, the analysis was restricted to data with silicon microvertex detector information (taken in 1991 and 1992), corresponding to an integrated luminosity of $30 \mathrm{pb}^{-1}$.

The kinematics of the Higgs boson signal varies rapidly with $m_{\mathrm{A}^{0}}$. While for a heavy $\mathrm{A}^{0}$ boson it is possible to resolve individual $b$ jets, for a lighter $A^{0}$, with mass close to the $b \bar{b}$ threshold, this is not always possible. On the other hand, light $\mathrm{A}^{0}$ bosons (which give rise to more collimated jets) can be reconstructed with higher accuracy than heavy $\mathrm{A}^{0}$ bosons. Therefore, in order to have a high selection efficiency over the whole relevant range of $m_{\mathrm{A}^{0}}$, we use two slightly different sets of kinematic cuts, one for the low mass part and one for the high mass part. The sensitivities of the two selections merge at $m_{\mathrm{A}^{0}} \approx 15 \mathrm{GeV} / \mathrm{c}^{2}$.

A preselection common to both $m_{\mathrm{A}^{0}}$ regions was first applied, requiring at least three jets, reconstructed by the JADE jet finding algorithm [34] with a mass resolution parameter $y_{c u t}=0.07$. The jet finder was applied to charged tracks and electromagnetic clusters which satisfied the standard quality requirements. In the few cases where more than three jets were found, the events were forced into a three jet pattern by increasing the value of $y_{c u t}$. All three jets were required to have their momentum vector pointing away from the beam direction, $(|\cos \theta|<0.9)$, and to contain at least three charged tracks. The four-momenta were determined using a global energy correction algorithm (GCE, see e.g. 
Figure 4
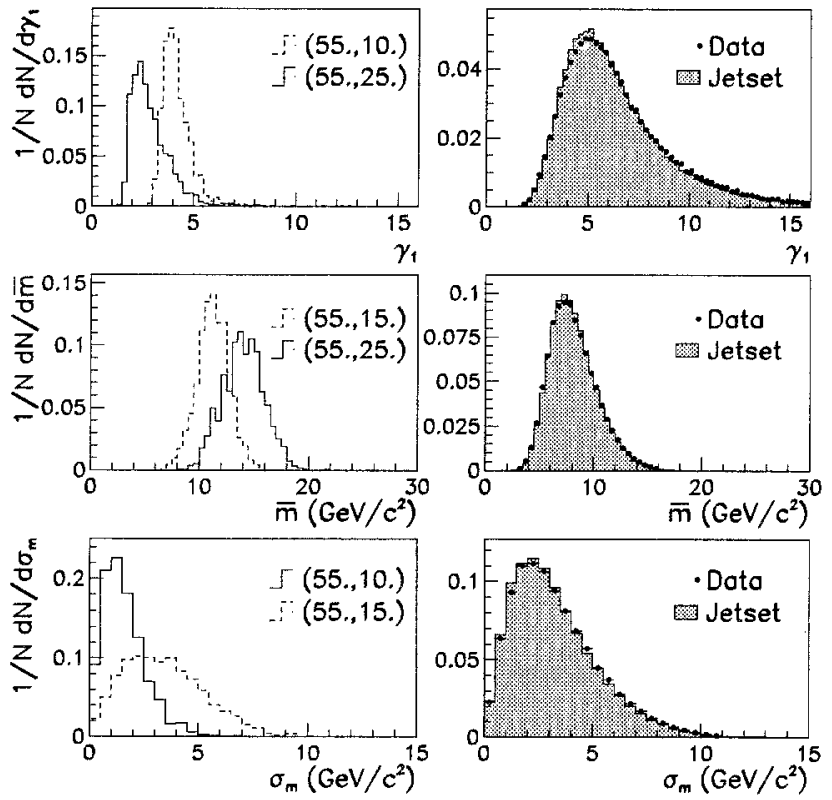

Fig. 4. Search for the $3(\mathrm{~b} \bar{b})$ final state [36]: kinematic distributions on which the selection cuts are based (see text). Left hand side: Higgs boson signal simulation for representative values of $\left(m_{h^{0}}, m_{\mathrm{A}^{0}}\right)$ (in $\left.\mathrm{GeV} / \mathrm{c}^{2}\right)$; right hand side: the data distributions compared to those of the QCD Monte Carlo, JETSET 7.3 [35]

[33]) which improves the measurement of jet four-vectors by avoiding the problem of double-counting the energy of charged tracks with associated electromagnetic clusters.

First it was required that all three jets have a moderate relativistic boost, $\gamma=E / m c^{2}$, less than 4 . The distribution of the highest of the three boost values is shown in the uppermost part of Fig. 4. In the high mass search we further required that the average value of the three jet masses be larger than $12 \mathrm{GeV} / \mathrm{c}^{2}$. As can be seen in Fig. 4, in the case of a heavy $\mathrm{A}^{0}$ boson the average jet mass is significantly lower than $m_{\mathrm{A}^{0}}$. While part of the difference is accounted for by the energy carried away by neutrinos the main reason is kinematical: since for large $m_{\mathrm{A}^{0}}$ the three $\mathrm{A}^{0}$ bosons have low momenta, the two $b$ jets are emitted at large angle. It is therefore rather difficult to correctly assign them to the parent $\mathrm{A}^{0}$ boson and the resulting average jet mass deviates from $m_{\mathrm{A}^{0}}$. In the low mass region, where the $\mathrm{A}^{0}$ bosons are correctly reconstructed, it is more efficient to require that the three jets have the same mass within the experimental resolution, by requesting that the average spread of the mass differences defined by their standard deviation, $\sigma_{m}$, be smaller than $4 \mathrm{GeV} / \mathrm{c}^{2}$. This distribution is also shown in Fig. 4.

The final selection is based on tagging $b$-flavoured hadrons which are about 10 times more abundant in the Higgs boson signal than in the QCD background. We reconstructed displaced secondary vertices, in the $r \phi$ plane (the plane perpendicular to the beam axis), from the decay of $b$ hadrons and used the normalised decay length, $l / \sigma_{l}$, where $\sigma_{l}$ is the measurement error associated with the measured decay length, $l$. (Note that for small decay lengths the value of $l$ can become negative due to the finite spatial resolution of the detector.) Since the aim was to identify $b$ hadrons within
Figure 5
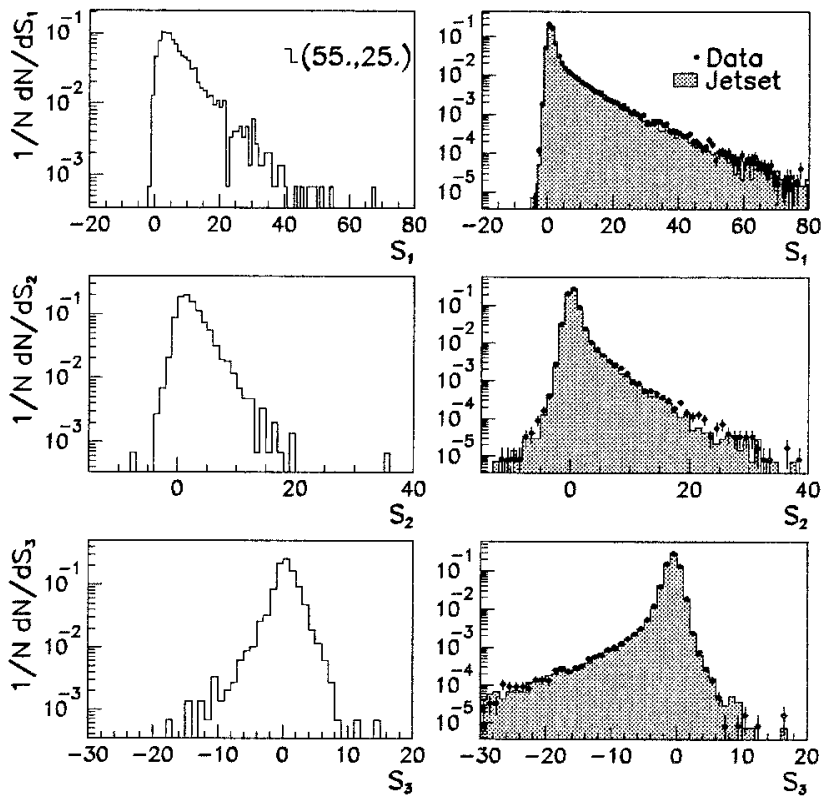

Fig. 5. Search for the 3(b) final state [36]: distributions of the three largest vertex significances, $S=l / \sigma_{l}$, in decreasing order $\left(S_{1}>S_{2}>S_{3}\right)$. Left hand side: Higgs boson signal with $m_{\mathrm{h}^{0}}=55 \mathrm{GeV} / \mathrm{c}^{2}$ and $m_{\mathrm{A}^{0}}=25$ $\mathrm{GeV} / \mathrm{c}^{2}$; right hand side: the data distributions compared to those of the QCD Monte Carlo, JETSET 7.3 [35]. Note the logarithmic scale of the vertical axis

individual $b$ jets, the events were reconstructed again, this time using the jet finding algorithm with a higher jet resolving power, $y_{c u t}=0.015$. The selection required at least three jets containing significantly displaced secondary vertices, with $l / \sigma_{l}>2$. The distributions for the three highest values of $S=l / \sigma_{l}$ are shown, in decreasing order, in Fig. 5 . The plots on the right demonstrate that the Monte Carlo simulation is able to reproduce accurately the data distributions in this important variable ${ }^{3}$. The difference between the signal and the QCD background is most pronounced for the third-largest $l / \sigma_{l}$, since for a Higgs boson decay the third displaced vertex would arise from a genuine $b$ hadron while for the $b \bar{b}+$ gluon background it is likely to be the result of the finite tracking resolution.

After all cuts, there remain 4225 data events in the low mass / high mass search. The background simulation predicts $(48.6 \pm 6.6 \pm 7.8) /(21.2 \pm 4.3 \pm 4.7)$ events, the first error being statistical and the second systematic. The systematic errors on the background prediction have been studied extensively [36]. The main contributions come from detector effects which reflect themselves in the relevant kinematic distributions and in the $l / \sigma_{l}$ distributions. Concerning the kinematic selection, the largest error, $15 \%$, arises from the cut on the average of the three jet masses, in the high mass selection. Concerning the selection based on the $l / \sigma_{l}$ distribution, modelling of the spatial resolution of the silicon microvertex detector results in an uncertainty of $14 \%$, while uncertainties in the hardness of $b$ quark fragmentation introduce an error of $7 \%$. Summing all systematic uncertainties

\footnotetext{
${ }^{3}$ In the Monte Carlo samples, the differences between reconstructed and generated track parameters, relevant to the determination of $l / \sigma_{l}$, had to be scaled by a factor 1.3 , to take into account detector misalignments which were not explicitly included in the event simulation
} 
in quadrature, one obtains $16 \% / 22 \%$ for the low mass / high mass analysis.

The detection efficiencies for the signal vary between $6 \%$ and $11 \%$. The relative systematic uncertainty is always smaller than $5 \%$. The total relative error, statistical and systematic combined, is in all cases smaller than $12 \%$. The domain excluded by this search at the $95 \% \mathrm{CL}$ is indicated by the dashed line labeled $\mathrm{C}$ in Fig. 3.

\section{The domain $m_{\mathrm{A}^{0}}<2 m_{\tau}$}

We now discuss the narrow band of the MSSM parameter space with $m_{\mathrm{A}^{0}}<2 m_{\tau}$ which was not fully excluded by the previous searches, as seen in Fig. 3. Here, the problems are similar to those which affected the low- $m_{\mathrm{h}^{0}}$ region and are due to the long lifetime and uncertain branching ratios of the $\mathrm{A}^{0}$ boson. In the following we discuss several new searches which were designed to cope with these difficulties.

\subsection{Searches in the domain $m_{\mathrm{A}^{0}}<2 m_{\mu}$}

One first considers the process $Z^{0} \rightarrow h^{0} A^{0} \rightarrow\left(A^{0} A^{0}\right) A^{0}$. Depending on the $A^{0}$ lifetime, this process gives either fully visible three-jet events, or partially visible two-jet or monojet events. Each of the jets is expected to have a small invariant mass $\left(\approx m_{\mathrm{A}^{0}}\right)$ and to be composed of either two charged tracks $\left(\mathrm{A}^{0} \rightarrow \mathrm{e}^{+} \mathrm{e}^{-}\right)$with a common vertex well separated from the primary $\mathrm{e}^{+} \mathrm{e}^{-}$interaction point, or of neutral electromagnetic energy clusters $\left(\mathrm{A}^{0} \rightarrow \gamma \gamma\right)$. In both cases the signal should be clearly visible in the electromagnetic calorimeter provided that the $\mathrm{A}^{0}$ boson decays before or inside this detector.

A search for such events was carried out using a data sample of $\approx 47 \mathrm{pb}^{-1}$ integrated luminosity. After applying the standard criteria to select well measured charged tracks and electromagnetic clusters we required:

Less than $5 \mathrm{GeV}$ energy deposited in the hadron calorimeter. Less than $2 \mathrm{GeV}$ energy deposited in the forward detector. $|\cos \theta|<0.95, \theta$ being the polar angle of the total momentum vector of the event.

No track recognised explicitly as a muon. ${ }^{4}$,

Not more than 3 jets.

The jet finder was used with a mass cutoff of $6 \mathrm{GeV} / \mathrm{c}^{2}\left(y_{\text {cut }}\right.$ $\approx 0.004$ ). Each of the jets was required to have a mass less than $2 \mathrm{GeV} / \mathrm{c}^{2}$, more than $20 \mathrm{GeV}$ energy in the electromagnetic calorimeter, a polar angle satisfying $|\cos \theta|<0.95$ and zero total electric charge. In the case of two-jet events one also required an acoplanarity angle greater than 10 degrees while in the case of monojet events the polar angle of the jet momentum was required to satisfy $|\cos \theta|<0.75$ and the transverse momentum to exceed $5 \mathrm{GeV} / \mathrm{c}$. This selection left 46 events in the data, 38 three-jet, 8 two-jet and 0 monojet events.

The background was studied using large samples of simulated $\mathrm{Z}^{0} \rightarrow \mathrm{q} \overline{\mathrm{q}}(+$ gluons $+\gamma \ldots), \mathrm{e}^{+} \mathrm{e}^{-} \rightarrow \gamma \gamma(\gamma), \nu \bar{\nu} \gamma(\gamma)$

\footnotetext{
${ }^{4}$ The identification of muons relies mainly in geometrical matching between charged tracks measured in the central detector and track segments reconstructed in the outer muon chambers (see e.g. Ref. [37])
}

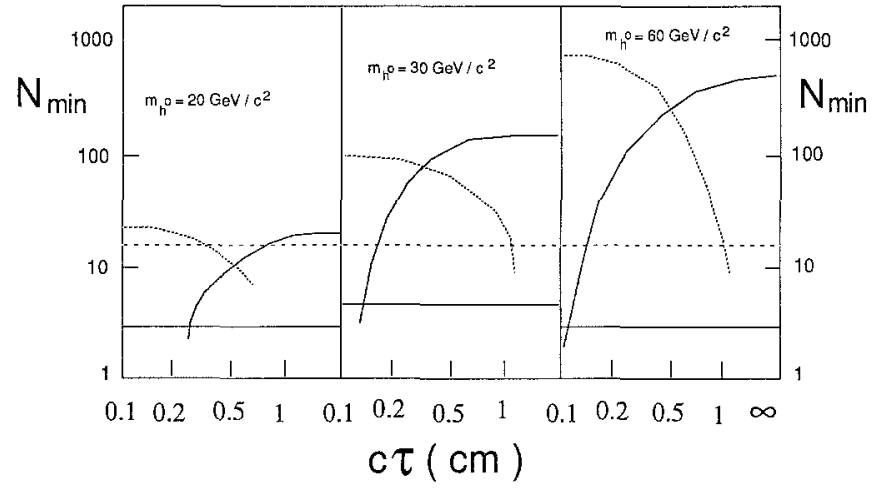

Fig. 6. Searches in the domain $m_{\mathrm{A}^{0}}<2 m_{\mu}$ : the smallest number of events, $\mathscr{N}_{\text {min }}$, for the signal, as a function of the decay length, $\mathrm{c} \tau$, of the $\mathrm{A}^{0}$ boson for fixed values of $m_{\mathrm{h}}$, compared to the $95 \% \mathrm{CL}$ upper limit (horizontal lines) derived from the data. Dashed lines: search for the $3\left(\mathrm{~A}^{0} \rightarrow\right.$ $\left.\mathrm{e}^{+} \mathrm{e}^{-}, \gamma \gamma\right)$ final state. Full lines: search for the $\mathrm{h}^{0}(\rightarrow \mathrm{f}) \mathrm{A}^{0}(\rightarrow$ invisible $)$ final state. The two searches together exclude $m_{\mathrm{h} 0} \geq 20 \mathrm{GeV} / \mathrm{c}^{2}$ for all values of the $\mathrm{A}^{0}$ boson lifetime and thus for all values of $m_{\mathrm{A}^{0}} \lesssim 200$ $\mathrm{MeV} / \mathrm{c}^{2}$

and $\ell^{+} \ell^{-} \gamma(\gamma)$ events. The remaining background was $51 \pm 6$ events of which $45 \pm 5$ were three-jet, $2 \pm 2$ two-jet and $4 \pm 4$ monojet events, consistent with the observed data.

The detection efficiencies for the Higgs boson signal were determined separately for the cases where all three $\mathrm{A}^{0}$ bosons decayed to $\mathrm{e}^{+} \mathrm{e}^{-}$and where one, two or three $\mathrm{A}^{0}$ bosons decayed to $\gamma \gamma$. The above selection cuts were designed to be decay-mode independent; nevertheless, the track reconstruction efficiency of the detector and the requirement on the total jet charge introduce a bias, disfavouring the $\mathrm{e}^{+} \mathrm{e}^{-}$final states. In order to obtain results which are valid irrespective of the ratio $\operatorname{Br}\left(\mathrm{A}^{0} \rightarrow \mathrm{e}^{+} \mathrm{e}^{-}\right) / \operatorname{Br}\left(\mathrm{A}^{0} \rightarrow \gamma \gamma\right)$, we used only the efficiencies obtained for the case when all three $\mathrm{A}^{0}$ bosons decay into $\mathrm{e}^{+} \mathrm{e}^{-}$. For $m_{\mathrm{A}^{0}}=25,50,100$ and $200 \mathrm{MeV} / \mathrm{c}^{2}$ we obtain detection efficiencies of $10 \%$, $13 \%, 24 \%$ and $30 \%$, respectively, independent of $m_{\mathrm{h}^{0}}$.

The smallest number of expected Higgs boson events, $\mathscr{N}_{\text {min }}$, (dashed curves) is compared in Fig. 6 to the $95 \%$ CL upper limit obtained from the data (dashed horizontal line). The horizontal scale represents the decay length, $c \tau$, of the $\mathrm{A}^{0}$ boson. In calculating the detection efficiency for the Higgs boson signal one has to take into account the probability, $\mathscr{P}$, as a function of $m_{\mathrm{A}^{0}}$, that the $\mathrm{A}^{0}$ boson escapes detection due to its long lifetime. This calculation took into account corrections due to heavy particles, allowing large variations for the top quark mass, the scalar-top quark mass and the chargino mass. For $m_{\mathrm{A}^{0}}=20,50$ and $100 \mathrm{MeV} / \mathrm{c}^{2}$ we obtain $\mathscr{P}=0.9,0.5$ and 0.05 , respectively, independent of $m_{\mathrm{h}^{0}}$.

The absence of a signal in this search could result from the long lifetime of a very light $\mathrm{A}^{0}$ boson. To address this possibility we considered the process $Z^{0} \rightarrow h^{0} A^{0}$ with the $h^{0}$ boson decaying into a pair of fermions. (The detection efficiency for the same process with $h^{0}$ decaying into $A^{0} A^{0}$ was set to zero.) Since in this case the $A^{0}$ boson was assumed to escape detection, the relevant event topology is identical to that of the process $\mathrm{Z}^{0} \rightarrow \mathrm{h}^{0} \mathrm{Z}^{*} \rightarrow \mathrm{f} f \bar{\nu}$ discussed in Sect. $4.1\left(\mathrm{Z}^{*} \rightarrow \nu \bar{\nu}\right.$ channel). That search (which had detection efficiencies of $\approx 40 \%$ and was based on a data sample of $\approx 75 \mathrm{pb}^{-1}$ [5]) is fully applicable to the present case. 
In calculating the detection efficiency, the probability, $\mathscr{P}$, for the $\mathrm{A}^{0}$ boson to escape detection has been taken into account. The smallest number of events, $\mathscr{N}_{\text {min }}$, expected from the Higgs boson signal, as a function of the decay length of the $\mathrm{A}^{0}$ boson, $\mathrm{c} \tau$, and for fixed $m_{\mathrm{h}^{0}}$, is compared in Fig. 6 to the $95 \% \mathrm{CL}$ upper limit obtained from the data (solid curve and solid horizontal line). Note, in the figure for $m_{\mathrm{h}^{0}}=30$ $\mathrm{GeV} / \mathrm{c}^{2}$, that the latter has been increased from 3 to 4.7 to take into account one observed event.

The present search completes the exclusion of a very light $\mathrm{A}^{0}$ boson with $m_{A^{0}}<2 m_{\mu}$ (which corresponds to $\mathrm{c} \tau \gtrsim 0.1 \mathrm{~cm}$ ), independent of its lifetime, for all values of $m_{\mathrm{h}^{0}}$ compatible with the MSSM.

\subsection{Search in the domain $2 m_{\mu}<m_{\mathrm{A}^{0}}<2 m_{\tau}$}

To cover this range of light $\mathrm{A}^{0}$ bosons one searched again for the process $Z^{0} \rightarrow h^{0} A^{0} \rightarrow\left(A^{0} A^{0}\right) A^{0}$ and examined a variety of possible $A^{0}$ decays. A typical example is the decay channel $\mathrm{A}^{0} \rightarrow \pi^{+} \pi^{-} \pi^{0}$. In total 17 decay modes were considered which included charged and neutral hadronic resonances $(\eta$, $\rho, \omega, \ldots)$, electrons, muons and fragmenting quarks. The selection criteria were designed to be insensitive to specific final states.

Independent of the decay mode, the Higgs boson signature consists of three well collimated jets with low mass and low multiplicity. After applying the standard selection to charged tracks and electromagnetic clusters we required:

The polar angle of the total momentum vector of the event to satisfy $|\cos \theta|<0.9$.

The event thrust to be less than 0.97 .

Three jets.

The jet finding algorithm was used with a mass cutoff parameter of $8 \mathrm{GeV} / \mathrm{c}^{2}$ (which corresponds to $y_{c u t} \approx 0.008$ ). Each of the three jets was required to have a polar angle satisfying $\left|\cos \theta_{j}\right|<0.9$, an invariant mass less than $7 \mathrm{GeV} / \mathrm{c}^{2}$ and energy larger than $15 \mathrm{GeV}$. Narrow collimation of the jets was imposed by requiring that at least $75 \%$ of the jet energy be contained in a cone of 15 degrees half-angle, centered on the jet axis.

A specific cut was applied against the process $Z^{0} \rightarrow$ $\ell^{+} \ell^{-} \gamma$. Events having two jets with a single charged track each and an angle greater than 90 degrees between them were removed if the third jet did not contain charged tracks.

Applied to a data sample of $\approx 22 \mathrm{pb}^{-1}$ this selection retained 1369 events while the simulation of the background resulted in $1296 \pm 38$ events. The largest contribution to the background comes from $Z^{0} \rightarrow q \bar{q}$ and smaller contributions from $Z^{0} \rightarrow \tau^{+} \tau^{-}, \mu^{+} \mu^{-} \gamma$ and $\mathrm{e}^{+} \mathrm{e}^{-} \gamma$.

Considering the decay $\mathrm{A}^{0} \rightarrow \pi^{+} \pi^{-} \pi^{0}$, the detection efficiency varies, for $m_{\mathrm{h}^{0}}$ between 30 and $65 \mathrm{GeV} / \mathrm{c}^{2}$ and $m_{\mathrm{A}^{0}}$ between 0.5 and $5 \mathrm{GeV} / \mathrm{c}^{2}$, from $13 \%$ to $65 \%$. For fixed $m_{\mathrm{A}^{0}}$ it increased with increasing $m_{\mathrm{h}^{0}}$ and for fixed $m_{\mathrm{h}^{0}}$ it decreased with increasing $m_{\mathrm{A}^{0}}$. For example, for $m_{\mathrm{h}^{0}}=50$ $\mathrm{GeV} / \mathrm{c}^{2}$ and $m_{\mathrm{A}^{0}}=0.5,3,5$ and $5.5 \mathrm{GeV} / \mathrm{c}^{2}$, one obtains $59 \%, 60 \%, 35 \%$ and $12 \%$, respectively. The study of the other decay modes gave similar efficiencies, with a spread of $\pm 5 \%$. To be decay-mode independent we used the smallest of these values.

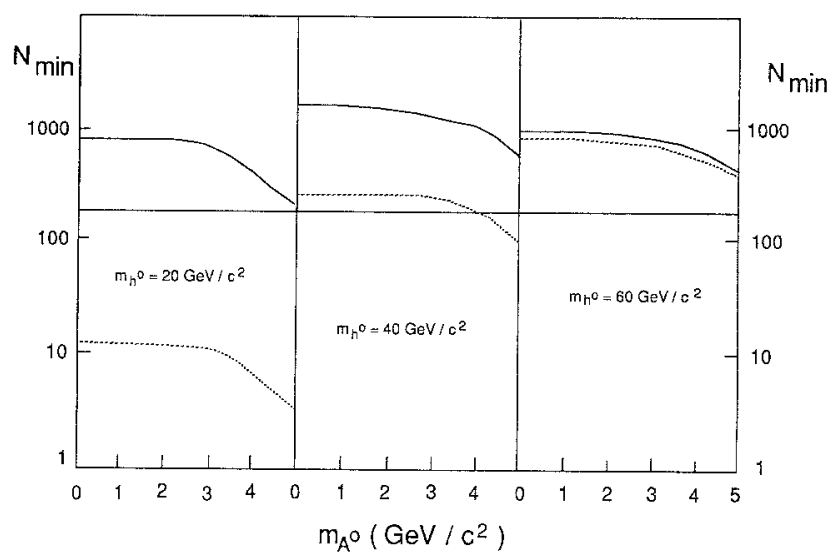

Fig. 7. Search in the domain $2 m_{\mu}<m_{\mathrm{A}^{0}}<2 m_{\tau}$ for $3 \mathrm{~A}^{0}$ final states: the smallest number of events, $\mathcal{A}_{\text {min }}$, for the signal, as a function of $m_{\mathrm{A}^{0}}$, for fixed values of $m_{\mathrm{h}}$, compared to the $95 \% \mathrm{CL}$ upper limit (horizontal lines) derived from the data. Dashed curves: this search alone; full curves: this search together with constraints from the SM Higgs boson searches and from $\Gamma_{Z^{0}}$

Figure 7 shows the minimal number of events, $\mathscr{N}_{\text {min }}$, expected for the Higgs boson signal, as a function of $m_{\mathrm{A}^{0}}$ and for fixed values of $m_{\mathrm{h}^{0}}$. It is compared to the $95 \% \mathrm{CL}$ upper limit obtained from the data (horizontal line). Even if this search were to be used alone (dashed curves), it would exclude the gap, defined by $2 m_{\mu}<m_{\mathrm{A}^{0}}<2 m_{\tau}$ and $m_{\mathrm{h}^{0}}>$ $46 \mathrm{GeV} / \mathrm{c}^{2}$, not excluded in Fig. 3. In conjunction with all other searches it starts to be effective already at $m_{\mathrm{h}^{0}}=20$ $\mathrm{GeV} / \mathrm{c}^{2}$ and becomes more significant towards higher $m_{\mathrm{h}^{0}}$, as indicated by the solid curves in Fig. 7 .

\section{Summary: MSSM}

Combining all the searches for the processes $\mathrm{Z}^{0} \rightarrow \mathrm{h}^{0} \mathrm{Z}^{*}$ and $Z^{0} \rightarrow h^{0} A^{0}$, we exclude, at the $95 \% \mathrm{CL}$, the existence of the $\mathrm{h}^{0}$ and $\mathrm{A}^{0}$ bosons over most of the MSSM parameter space which can be accessed at the current LEP energy. We have analysed a wide range of Higgs boson final states and also used the constraint from the precise LEP measurements of the $\mathrm{Z}^{0}$ decay width. Decay-mode independent analyses were devised for the cases in which either $m_{\mathrm{h}^{0}}$ or $m_{\mathrm{A}^{0}}$ were less than $2 m_{\tau}$. In these regions the $\mathrm{h}^{0}$ and $\mathrm{A}^{0}$ boson decay modes are not reliably predicted. Moreover, very light Higgs bosons have a long lifetime and may escape the detector.

The $95 \% \mathrm{CL}$ exclusion is given by the shaded area in Fig. 8. It is valid in the MSSM context as formulated in Ref. [14] for $\tan \beta \geq 1$. In evaluating the one-loop radiative corrections we assumed $m_{\mathrm{t}}<200 \mathrm{GeV} / \mathrm{c}^{2}$ and $m_{\mathrm{S}}<1$ $\mathrm{TeV} / \mathrm{c}^{2}$. The results imply the following lower limits: $m_{\mathrm{h}^{0}}=$ $44.5 \mathrm{GeV} / \mathrm{c}^{2}$ (at $m_{\mathrm{A}^{0}}=45.2 \mathrm{GeV} / \mathrm{c}^{2}$ ) and $m_{\mathrm{A}^{0}}=24.3$ $\mathrm{GeV} / \mathrm{c}^{2}$ (at $m_{\mathrm{h}^{0}}=61.0 \mathrm{GeV} / \mathrm{c}^{2}$ ). These numbers slightly improve those published by other LEP experiments [38]. The first limit is determined predominantly by the search for the $\mathrm{Z}^{0} \rightarrow \mathrm{h}^{0} \mathrm{~A}^{0} \rightarrow \tau^{+} \tau^{-} \mathrm{q} \overline{\mathrm{q}}$ final state and is saturated by the kinematic bound, $m_{\mathrm{b}^{0}}+m_{\mathrm{A}^{0}}=m_{\mathrm{Z}^{0}}$. The second limit is determined essentially by the search for the $Z^{0} \rightarrow h^{0} A^{0} \rightarrow$ $\left(\mathrm{A}^{0} \mathrm{~A}^{0}\right) \mathrm{A}^{0} \rightarrow 3(\mathrm{~b} \overline{\mathrm{b}})$ final state. With higher luminosity this limit can, in principle, be slightly improved by pursuing the search for the $\mathrm{h}^{0}$ boson in the "bremsstrahlung" channel.

In order to assess the overall robustness of our exclusions, we decreased all detection efficiencies simultaneously. 


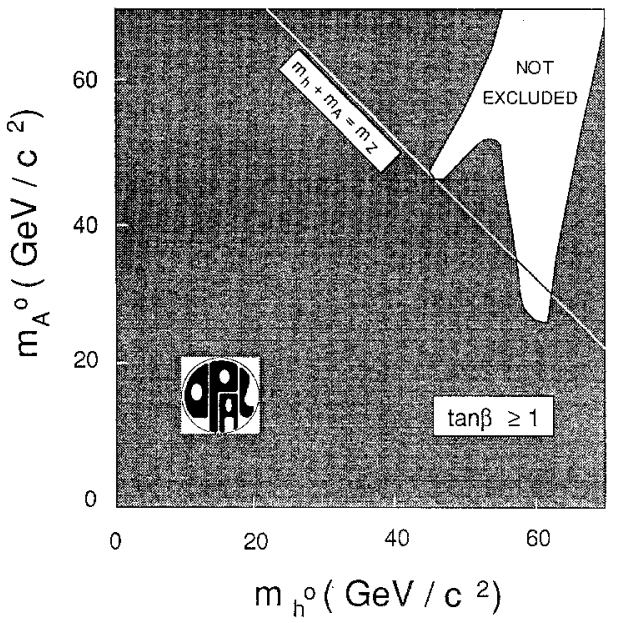

Fig. 8. The OPAL exclusion ( $95 \% \mathrm{CL}$ ) in the MSSM parameter space for $\tan \beta \geq 1$. For the calculation of radiative corrections, $m_{\mathrm{t}} \leq 200$ $\mathrm{GeV} / \mathrm{c}^{2}$ and $m_{\mathrm{S}} \leq 1 \mathrm{TeV} / \mathrm{c}^{2}$ were assumed. The unshaded area is not excluded

For a decrease by a factor 0.6 the exclusion was still continuous and the $95 \% \mathrm{CL}$ lower limits on $m_{\mathrm{h}^{0}}$ and $m_{\mathrm{A}^{0}}$ changed to $44.0 \mathrm{GeV} / \mathrm{c}^{2}$ and $21.5 \mathrm{GeV} / \mathrm{c}^{2}$, respectively.

The excluded region depends to some extent on the calculation of one-loop radiative corrections and, in particular, on the maximum value, $\epsilon_{\max }$, of the parameter $\epsilon$ defined in Eq. (4). In order to quantify the model-dependence of our result, we have varied $\epsilon_{\max }$ and determined the corresponding 95\% CL limits. These are presented in Fig.9, where the shaded areas represent exclusions for various values of $\epsilon_{\max }$. The areas correspond, from inside to outside, to $\epsilon_{\max }=0.5 \epsilon_{\max }^{o}, \epsilon_{\max }^{o}$ and $2 \epsilon_{\max }^{o}$. Here, $\epsilon_{\max }^{o}$ is our standard value, corresponding to $m_{\mathrm{t}}=200 \mathrm{GeV} / \mathrm{c}^{2}$ and $m_{\mathrm{S}}=1 \mathrm{TeV} / \mathrm{c}^{2}$. An increase of $\epsilon_{\max }$ with respect to $\epsilon_{\max }^{0}$ by a factor 2 corresponds to increasing the limit on $m_{\mathrm{t}}$ to $235 \mathrm{GeV} / \mathrm{c}^{2}$ or that on $m_{\mathrm{S}}$ to approximately $7 \mathrm{TeV} / \mathrm{c}^{2}$.

Although the assumption $\tan \beta \geq 1$ is theoretically favoured, the possibility of $\tan \beta<1$ cannot be ignored (see e.g. Refs. [13, 15, 39, 40]). The assumption $\tan \beta<$ 1 enlarges the MSSM parameter space and modifies the Higgs boson couplings. The branching fractions into up-type fermion final states $\left(e . g . \mathrm{h}^{0}, \mathrm{~A}^{0} \rightarrow \mathrm{c} \overline{\mathrm{c}}\right)$ increase and those into down-type final states (e.g. $\left.\mathrm{h}^{0}, \mathrm{~A}^{0} \rightarrow \mathrm{b} \overline{\mathrm{b}}, \tau^{+} \tau^{-}\right)$decrease. Consequently, searches which rely either on tagging b-flavoured hadrons or on identifying $\tau^{+} \tau^{-}$pairs are less effective. Figure 10 shows exclusion contours for cases with $\tan \beta<1$. Arguments based on the perturbative character of the electroweak theory only [39] give $\tan \beta>0.2$. On the other hand, if one assumes that the MSSM remains valid up to the Planck scale, one obtains the relation [40] $\sin \beta=m_{\mathrm{t}}[$ $\left.\mathrm{GeV} / \mathrm{c}^{2}\right] / 200$ which implies $\tan \beta>0.85$ for $m_{\mathrm{t}}>131$ $\mathrm{GeV} / \mathrm{c}^{2}[26]$.

\section{Limits valid for general two-doublet models}

The searches described in the previous sections have also been used to obtain a limit in the framework of general models with two Higgs field doublets [12]. In such models the cross sections expressed by Eqs. (1) and (2) are valid, but the Higgs boson decay branching ratios are not predicted.

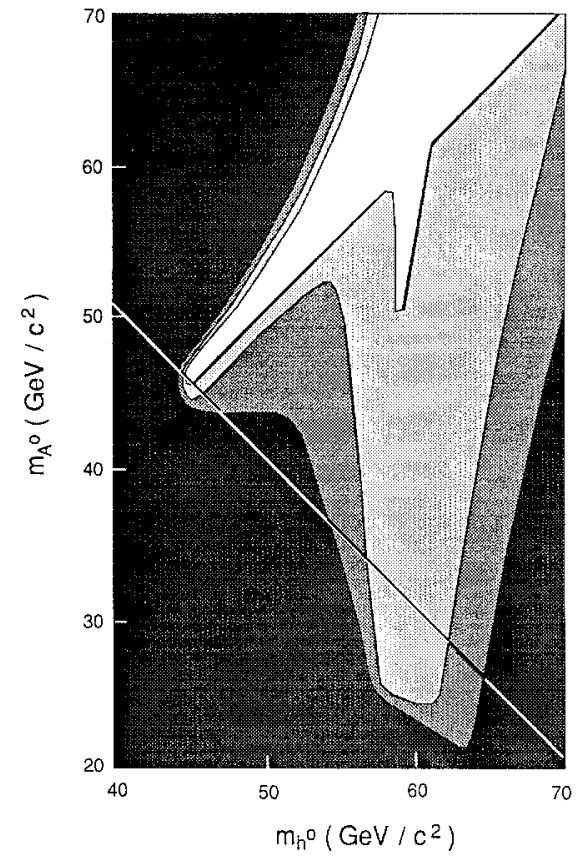

Fig. 9. Excluded regions in the $\left(m_{\mathrm{h}^{0}}, m_{\mathrm{A}^{0}}\right)$ plane $(\tan \beta \geq 1)$ and for various ranges, $0 \leq \epsilon \leq \epsilon_{\max }$, of the radiative corrections. The shadings correspond, starting from inside (blank) to outside, to $\epsilon_{\max }=0.5 \epsilon_{\max }^{0}, \epsilon_{\max }^{0}$ and $2 \epsilon_{\max }^{0}$, respectively, where $\epsilon_{\max }^{0}$ is our standard value of $\epsilon_{\max }$, which corresponds to $m_{\mathrm{t}}=200 \mathrm{GeV} / \mathrm{c}^{2}$ and $m_{\mathrm{S}}=1 \mathrm{TeV} / \mathrm{c}^{2}$

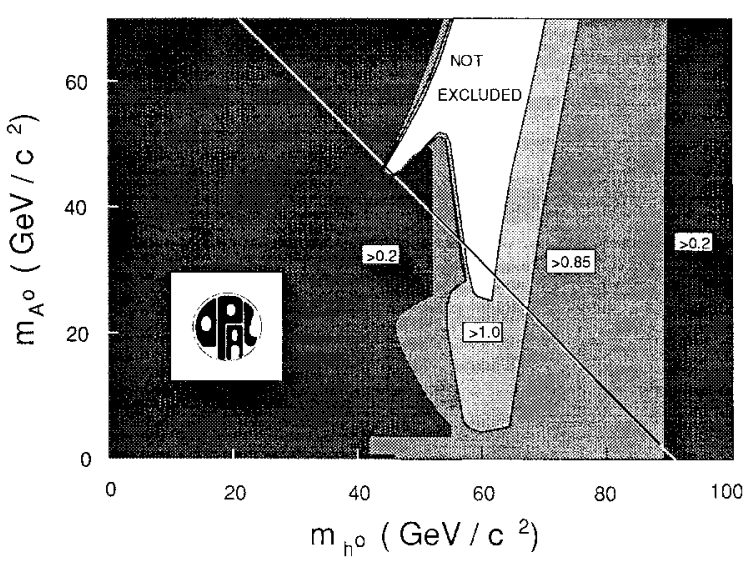

Fig. 10. Exclusions (95\% CL) in the MSSM parameter space for various ranges of $\tan \beta$ (indicated in the figure)

Points in the $\left(m_{\mathrm{h}^{0}}, m_{\mathrm{A}^{0}}\right)$ plane in which the sum of the upper bounds on $\cos ^{2}(\beta-\alpha)$ and $\sin ^{2}(\beta-\alpha)$ is less than 1 are excluded. The upper bound on $\cos ^{2}(\beta-\alpha)$ is derived from the measurement of the $Z^{0}$ width [Eq. (2)] and therefore is not affected by the uncertainty on the Higgs boson decay branching fractions. On the other hand, the upper bound on $\sin ^{2}(\beta-\alpha)$ is derived from the searches in the neutrino $\left(\mathrm{Z}^{0} \rightarrow \mathrm{h}^{0} \nu \bar{\nu}\right)$ and leptonic $\left(\mathrm{Z}^{0} \rightarrow \mathrm{h}^{0} \mathrm{e}^{+} \mathrm{e}^{-}, \mathrm{h}^{0} \mu^{+} \mu^{-}\right)$channels and depends on the Higgs boson decay modes.

In the case of a relatively light $\mathrm{h}^{0}$ boson $\left(m_{\mathrm{h}^{0}}<11.3\right.$ $\mathrm{GeV} / \mathrm{c}^{2}$ ) the decay-mode independent analysis has been used (Sect. 3 ) in conjunction with the constraint from the $Z^{0}$ width to exclude the dark area labeled A in Fig. 11. The modelindependence of the analysis allows the extension of the excluded region all the way down to $m_{\mathrm{A}^{0}}=0$. The constraint from the $\mathrm{Z}^{0}$ width becomes weaker for heavy $\mathrm{A}^{0}$ due to 


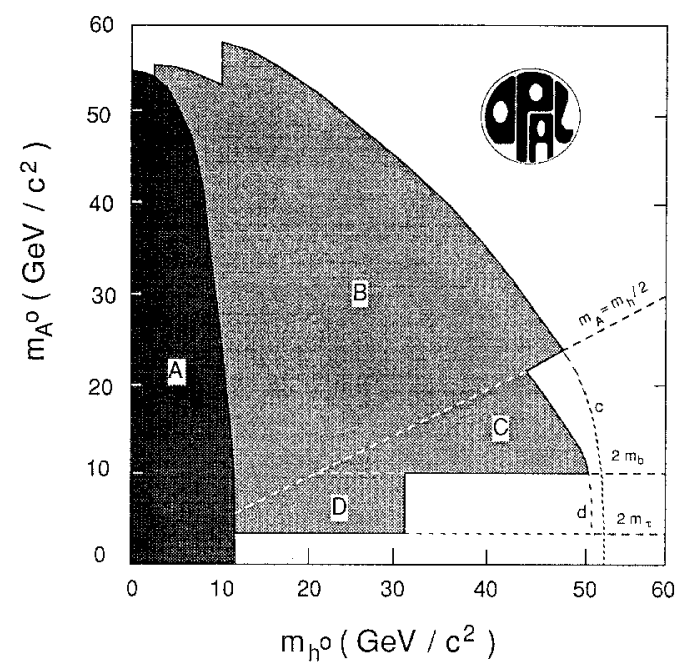

Fig. 11. Exclusions in the $\left(m_{\mathrm{h}^{0}}, m_{\mathrm{A}^{0}}\right)$ plane valid in general two-doublet Higgs models. The dark- and light-shaded areas together represent our $95 \%$ CL exclusion. More details are given in the text

the decrease of phase space $[Q \rightarrow 0$ in Eq. (2)], therefore limiting the excluded region from above.

For a heavier $\mathrm{h}^{0}$ boson the $\mathrm{Z}^{*} \rightarrow \nu \bar{\nu}$ and $\mathrm{Z}^{*} \rightarrow$ $\mathrm{e}^{+} \mathrm{e}^{-}, \mu^{+} \mu^{-}$channel analyses are used to derive the upper limit on $\sin ^{2}(\beta-\alpha)$. In evaluating the detection efficiency we have taken into account all possible decay modes and retained the one with the lowest efficiency.

In the region $m_{\mathrm{A}^{0}}>m_{\mathrm{h}^{0}} / 2$ the $\mathrm{h}^{0}$ boson decays to either a pair of quarks (b $\bar{b}$ or $c \bar{c})$ or $\tau$ leptons $\left(\tau^{+} \tau^{-}\right)$. Three cases are considered: In the first case, the Higgs scalar couples in an SM-like manner leading to hadronic final states in more than $90 \%$ of the cases. In the second case, the Higgs scalar couples only to down-type fermions (as in the SUSY case, with $\tan \beta \geq 1$ ). In such a case the ratio between $h^{0} \rightarrow \tau^{+} \tau^{-}$and $h^{0} \rightarrow b \bar{b}$ is practically model-independent and depends on the running-mass ratio only, resulting in a hadronic branching ratio of $92-94 \%$. In the third case, the Higgs scalar couples to up-type fermions only (as in the SUSY case, with $\tan \beta<1$ ), leading to a pure cc cinal state.

The searches in the neutrino and leptonic channels are not sensitive to differences between $c \bar{c}$ and $b \bar{b}$ final states. Therefore, the worst possible case - in which $92 \%$ of the decays are to hadronic final states - is used when deriving the upper bound on $\sin ^{2}(\beta-\alpha)$. This limit, together with the $Z^{0}$ width constraint leads to the exclusion of the grey area, labeled B in Fig. 11. The discontinuity at $m_{\mathrm{h}^{0}}=10$ $\mathrm{GeV} / \mathrm{c}^{2}\left(m_{\mathrm{A}^{0}} \approx 55 \mathrm{GeV} / \mathrm{c}^{2}\right)$ is due to the fact that our most recent analysis is not extended below $10 \mathrm{GeV} / \mathrm{c}^{2}$ where an earlier published analysis [41], based on a lower luminosity, is used.

In the region $2 m_{\mathrm{b}}<m_{\mathrm{A}^{0}}<m_{\mathrm{h}^{0}} / 2$, the lowest detection efficiency is obtained for the decay $h^{0} \rightarrow \mathrm{A}^{0} \mathrm{~A}^{0}$ where the $\mathrm{A}^{0}$ decays predominantly $(92 \%)$ to hadronic final states due to similar reasons as mentioned above. This leads to the exclusion of the region labeled $\mathrm{C}$ in Fig. 11. The dashed line labeled $\mathrm{c}$ shows the exclusion which would be obtained for models in which the $\mathrm{h}^{0} \rightarrow \mathrm{A}^{0} \mathrm{~A}^{0}$ decay would be suppressed.

Finally, in the region $2 m_{\tau}<m_{\mathrm{A}^{0}}<2 m_{\mathrm{b}}$ and below the line $m_{\mathrm{A}^{0}}=m_{\mathrm{h}^{0}} / 2$ one has to consider the decay $Z^{0} \rightarrow$ $\mathrm{h}^{0} \mathrm{~A}^{0} \rightarrow\left(\mathrm{A}^{0} \mathrm{~A}^{0}\right) \mathrm{A}^{0}$ with $\mathrm{A}^{0} \rightarrow \tau^{+} \tau^{-}$, since it gives the lowest detection efficiency. This results in the exclusion of the region marked D in Fig. 11. The dashed line labeled $d$ shows the exclusion which would be obtained for models in which the $\mathrm{A}^{0} \rightarrow \tau^{+} \tau^{-}$decay mode would be suppressed.

Since the branching fractions of a very light $\mathrm{A}^{0}$ boson $\left(m_{\mathrm{A}^{0}}<2 m_{\tau}\right)$ are uncertain, and following the procedure of taking the least favourable case, none of the above mentioned analyses is capable of excluding this region, except for $m_{\mathrm{h}^{0}}<11.3 \mathrm{GeV} / \mathrm{c}^{2}$ where the exclusion was obtained from the decay-mode independent analysis.

Acknowledgements. It is a pleasure to thank the SL Division for the efficient operation of the LEP accelerator, the precise information on the absolute energy, and their continuing close cooperation with our experimental group. In addition to the support staff at our own institutions we are pleased to acknowledge the Department of Energy, USA, National Science Foundation, USA, Texas National Research Laboratory Commission, USA, Particle Physics and Astronomy Research Council, UK, Natural Sciences and Engineering Research Council, Canada, Fussefeld Foundation, Israeli Ministry of Energy and Ministry of Science, Israel Science Foundation, administered by the Israel Academy of Science and Humanities, Minerva Gesellschaft, Japanese Ministry of Education, Science and Culture (the Monbusho) and a grant under the Monbusho International Science Research Program, German Israeli Bi-national Science Foundation (GIF), Direction des Sciences de la Matière du Commissariat à l'Energie Atomique, France, Bundesministerium für Forschung und Technologie, Germany, National Research Council of Canada, A.P. Sloan Foundation and Junta Nacional de Investigação Científica e Tecnológica, Portugal.

\section{References}

1. P. W. Higgs, Phys. Lett. 12 (1964) 132; F. Englert and R. Brout, Phys. Rev. Lett. 13 (1964) 321; G. S. Guralnik, C. R. Hagen, and T. W. B. Kibble, Phys. Rev. Lett. 13 (1964) 585

2. S. L. Glashow, J. Illiopoulos and L. Maiani, Phys. Rev. D2 (1970) 1285; S. Weinberg, Phys. Rev. Lett. 19 (1967) 1264; A. Salam, Elementary Particle Theory, ed. N. Svartholm (Almquist and Wiksells, Stockholm, 1969), p. 367; B. W. Lee, C. Quigg and G. B. Thacker, Phys. Rev. Lett. 38 (1977) 883; Phys. Rev. D16 (77) 1519

3. J. Bjorken, in Proceedings of the 1976 SLAC Summer Institute on Particle Physics, SLAC-198 (1977) 1

4. ALEPH Collaboration, D. Buskulic et al., Phys. Lett. B313 (1993) 299; DELPHI Collaboration, P. Abreu et al., CERN-PPE/94-46/Revised, submitted to Nucl. Phys. B; L3 Collaboration, O. Adriani et al., Phys. Lett. B303 (1993) 391

5. OPAL Collaboration, R. Akers et al., Phys. Lett. B327 (1994) 397

6. E. Gross and P. Yepes, Int. Journ. Mod. Phys. A, Vol. 8, No. 3 (1993) 407

7. G. Giacomelli and P. Giacomelli, La Rivista del Nuovo Cimento, Vol. 16 , No. 3

8. P. Janot, LAL Report 92-27 and also in Proceedings of the XXVII Rencontres de Moriond (1993), Savoie, France

9. D. Froidevaux, Z. Kunszt, J. Stirling et al., in Proceedings of the Large Hadron Collider Workshop, Aachen 1990, Report CERN 90-10; G. Altarelli, ibid.; D. Denegri, ibid.

10. $\mathrm{e}^{+} \mathrm{e}^{-}$Collisions at $500 \mathrm{GeV}$ : The Physics Potential, Proceedings of the Workshop Munich, Annecy, Hamburg, Feb. 4-Sept.3, 1991; Proceedings of the Workshop on Physics and Experiments with Linear $\mathrm{e}^{+} \mathrm{e}^{-}$ Colliders, Waikoloa, Hawaii, 26-30 April 1993

11. N. Cabibbo, L. Maiani, G. Parisi and R. Petronzio, Nucl. Phys. B158 (1979) 295; M. Chanowitz, M. Furmann and I. Hinchliffe, Phys. Lett. B78 (1978) 285; R. A. Flores and M. Sher, Phys. Rev. D27 (1983) 1679; M. Lindner, Z. Phys. C31 (1986) 295

12. Higgs search and New Physics, P.J. Franzini, P. Taxil et al., in $Z$ Physics at LEP I, Vol. 2, CERN 89-08 (1989); J. F. Gunion, H. E. Haber, G. L. Kane and S. Dawson, Higgs Hunter's Guide, AddisonWesley, Redwood City, CA (1990) and references therein 
13. P. H. Chankowski, S. Pokorski and J. Rosiek, Phys. Lett, B274 (1992) 191; Phys. Lett. B281 (1992) 100; Phys. Lett. B286 (1992) 307

14. Y. Okada, M. Yamaguchi and T. Yanagida, Prog. Theor. Phys. Lett. 85 (1991) 1; Phys. Lett. B262 (1991) 54; A. Yamada, Phys. Lett. B263 (1991) 233; J. Ellis, G. Ridolfi, F. Zwirner, Phys. Lett. B258 (1991) 167; Phys. Lett. B262 (1991) 477, and references therein; H. E. Haber and R. Hempfling, Phys. Rev. Lett. 66 (1991) 1815; A. Brignole and F. Zwirner, Phys. Lett. B299 (1993) 72

15. G. L. Kane et al., Phys. Rev. Lett. 70 (1993) 26862; UM-TH-93-24 (1993)

16. The LEP Collaborations: ALEPH, DELPHI, L3, OPAL and The LEP Electroweak Working Group, CERN-PPE/93-157 (August 1993).

17. M. Koratzinos: Electroweak results from LEP in Proceedings of the $\mathrm{La}$ Thuile Conference (1994), LEP preliminary results prepared for the La Thuile and Moriond Conferences

18. G. Altarelli, R. Barbieri and F. Caravaglios, Phys. Lett. B314 (1993) 357; R. Barbieri, M. Frigeni and F. Caravaglios, Phys. Lett. B279 (1992) 169

19. F. Abe et al., Phys. Rev. Lett. 73 (1994) 225; Fermilab-Pub-94/097-E (1994)

20. A. Brignole, J. Ellis, G. Ridolfi and F. Zwirner, Phys. Lett. B271 (1991) 123; H. E. Haber, R. Hempfling and Y. Nir, Phys. Rev. D46 (1992) 3015

21. OPAL Collaboration, K. Ahmet et al., Nucl. Instr. Meth. A305 (1991) 275

22. OPAL Collaboration, M. Z. Akrawy et al., Phys. Lett. B251 (1990) 211

23. OPAL Collaboration, P. D. Acton et al., Phys. Lett. B268 (1991) 122

24. R. Barbieri and M. Frigeni, Phys. Lett. B258 (1991) 395

25. A. Brignole et al., Phys. Lett. B271 (1991) 123

26. D0 Collaboration, S. Abachi et al., Phys. Rev. Lett. 72 (1994) 2138
27. P. Igo-Kemenes, in Proceedings of the Joint International LeptonPhoton Symposium and Europhysics Conference on High Energy Physics, Geneva, Switzerland, 25 July-1 August 1991

28. The Particle Data Group, Phys. Rev. D45 (1992) Part 2

29. O. Helene, Nucl. Instr. Meth. 212 (1983) 319

30. D. Schaile, Tests of the Electroweak Theory at LEP, CERN-PPE/93213, submitted to Fortschritte der Physik

31. OPAL Collaboration, M. Z. Akrawy, et al., Z. Phys. C49 (1991) 1. While the methods described are valid, some of the conclusions have been invalidated by the realisation of the importance of one-loop radiative corrections

32. F. A. Berends, P. H. Daverveldt and R. Kleiss, Nucl. Phys. B253 (1985) 421; J. Hilgart, R. Kleiss and F. Le Diberder, Comput. Phys. Commun. 75 (1993) 191

33. OPAL Collaboration, M. Z. Akrawy et al., Phys. Lett. B253 (1991) 511

34. W. Bartel et al., Z. Phys. C33 (1986) 23

35. T. Sjöstrand, Comp. Phys. Comm. 39 (1986) 347; T. Sjöstrand and M. Bengtsson, Comp. Phys. Comm. 43 (1987) 367; T. Sjöstrand, CERN$\mathrm{TH} / 6488 / 92$

36. P. Teixeira-Dias, Ph. D. Thesis (in preparation), Physikalisches Institut, Univ. Heidelberg, Germany

37. OPAL Collaboration, P. D. Acton et al., Phys. Lett. B276 (1992) 379

38. ALEPH Collaboration, D. Buskulic et al., Phys. Lett. B313 (1993) 312; DELPHI Collaboration, P. Abreu et al., Nucl. Phys. B373 (1992) 3; L3 Collaboration, O. Adriani et al., Z. Phys. C57 (1993) 355

39. V. Barger, J. Hewett and R. Phillips, Phys. Rev. D41 (1990) 3421

40. J. L. Lopez and D. V. Nanopoulos, Mod. Phys. Lett. A5 (1990) 1259

41. OPAL Collaboration, M. Z. Akrawy et al., Phys. Lett. B236 (1990) 224 